\title{
Inhibition of estrogen signaling in myeloid cells increases tumor immunity in melanoma
}

\author{
Binita Chakraborty, ${ }^{1}$ Jovita Byemerwa, ${ }^{1}$ Jonathan Shepherd, ${ }^{2}$ Corinne N. Haines, ${ }^{1}$ Robert Baldi, ${ }^{1}$ Weida Gong, ${ }^{2}$ Wen Liu, ${ }^{1}$ \\ Debarati Mukherjee, ${ }^{1}$ Sandeep Artham, ${ }^{1}$ Felicia Lim, ${ }^{1}$ Yeeun Bae, ${ }^{1}$ Olivia Brueckner, ${ }^{1}$ Kendall Tavares, ${ }^{1}$ Suzanne E. Wardell, ${ }^{1}$ \\ Brent A. Hanks, ${ }^{3}$ Charles M. Perou, ${ }^{2}$ Ching-Yi Chang, ${ }^{1}$ and Donald P. McDonnell ${ }^{1}$
}

'Department of Pharmacology and Cancer Biology, Duke University School of Medicine, Durham, North Carolina, USA. 'Lineberger Comprehensive Cancer Center, University of North Carolina at Chapel Hill, Chapel Hill, North Carolina, USA. ${ }^{3}$ Department of Medicine, Duke University School of Medicine, Durham, North Carolina, USA.

\begin{abstract}
Immune checkpoint blockade (ICB) therapies have significantly prolonged patient survival across multiple tumor types, particularly in melanoma. Interestingly, sex-specific differences in response to ICB have been observed, with males receiving a greater benefit from ICB than females, although the mechanism or mechanisms underlying this difference are unknown. Mining published transcriptomic data sets, we determined that the response to ICBs is influenced by the functionality of intratumoral macrophages. This puts into context our observation that estrogens (E2) working through the estrogen receptor $\alpha(E R \alpha)$ stimulated melanoma growth in murine models by skewing macrophage polarization toward an immune-suppressive state that promoted CD8+ $\mathrm{T}$ cell dysfunction and exhaustion and ICB resistance. This activity was not evident in mice harboring macrophage-specific depletion of ER $\alpha$, confirming a direct role for estrogen signaling within myeloid cells in establishing an immunosuppressed state. Inhibition of ERo using fulvestrant, a selective estrogen receptor downregulator (SERD), decreased tumor growth, stimulated adaptive immunity, and increased the antitumor efficacy of ICBs. Further, a gene signature that determines ER activity in macrophages predicted survival in patients with melanoma treated with ICB. These results highlight the importance of E2/ER signaling as a regulator of intratumoral macrophage polarization, an activity that can be therapeutically targeted to reverse immune suppression and increase ICB efficacy.
\end{abstract}

\section{Introduction}

Metastatic melanoma is one of the most aggressive, morbid cancers, with a median survival of 6 to 9 months (1). Whereas the development of MAPK pathway inhibitors and antibodies directed against immune checkpoints has significantly improved outcomes in this disease, de novo and acquired resistance to these therapies remains a major impediment to achieving a durable clinical response in most patients (2-5). Further, although complete responses to combination immune checkpoint blockade (ICB) therapies (anti-CTLA4 plus anti-programmed cell death 1 [ $\alpha$-CTLA4 plus $\alpha$-PD-1]) are achieved in approximately $20 \%$ of patients (6), the general toxicity- and immune-related adverse events seen in the majority of individuals who receive the existing combination therapies significantly limit their clinical use (7). Thus, strategies that increase the efficacy and/or reduce the toxicities associated with ICB would likely expand the clinical utility of existing drugs and ultimately improve long-term outcomes in this disease.

The classification of melanoma as a hormone-sensitive neoplasm remains controversial, and the importance of hormone-associated risk factors, such as pregnancy, menopausal status, hormone therapies, and the use of oral contraceptives, in the pathobiology of

Conflict of interest: The authors have declared that no conflict of interest exists. Copyright: () 2021, American Society for Clinical Investigation.

Submitted: May 14, 2021; Accepted: September 29, 2021; Published: December 1, 2021

Reference information: J Clin Invest. 2021;131(23):e151347.

https://doi.org/10.1172/JCl151347. this disease remains unclear (8-12). Although the potential effects of sex steroids on melanoma risk need to be assessed in large clinical studies, there already exists compelling evidence that the incidence of secondary melanoma is significantly lower in anti-estrogen-treated breast cancer patients than in the general population (13). Further, the results of a recently published meta-analysis revealed that the degree of benefit from ICB treatment in patients with melanoma, and in patients with non-small-cell lung cancer, is lower in women than in men (14). Considering these observations, we hypothesized that there are sex hormone-dependent baseline differences in the immune system that contribute to sex-specific differences in tumor immunity and ICB efficacy. Under normal physiological conditions and in some disease contexts, it has been demonstrated that female sex steroids that target the estrogen receptor (ER) affect the differentiation and function of both the humoral and adaptive immune systems (15). However, the extent to which estrogen action/signaling in the tumor-immune microenvironment impacts the growth of melanoma and if and how this signaling axis can be exploited for therapeutic benefit have not been established.

Estrogens mediate their physiological actions in cells through the classical nuclear ERs (ER $\alpha$ and ER $\beta$ ) and through the nonclassical GPCR GPER1 (also referred to as GPR30). A recent study by Natale et al. highlighted a tumor cell-intrinsic role for GPER1 in regulating melanocyte differentiation, thereby preventing melanoma cell proliferation. Further, a synergistic antitumor response was observed when GPER agonists were combined with immune checkpoint inhibitors (16). While anecdotal evidence exists 

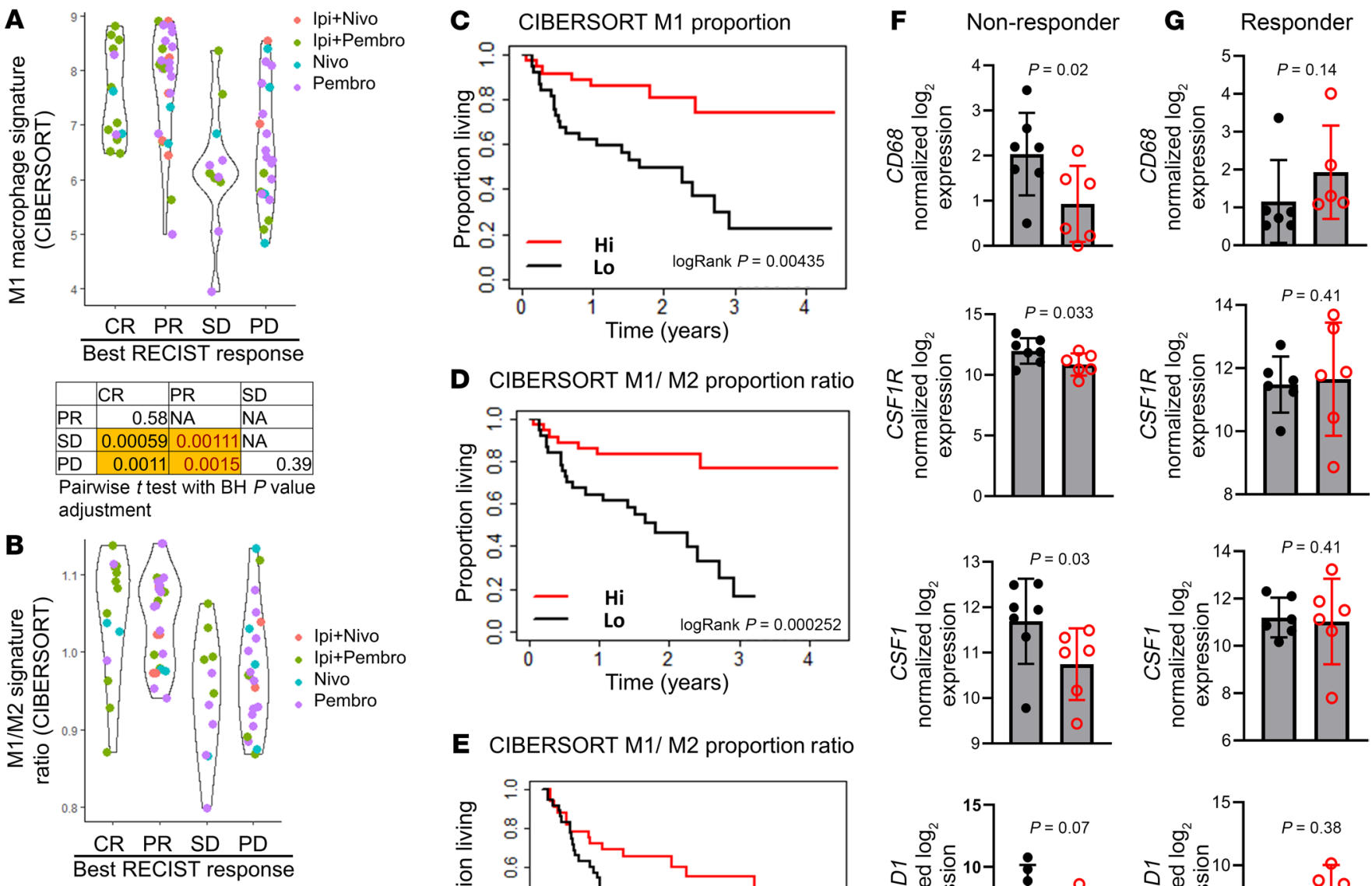

D CIBERSORT M1/M2 proportion ratio

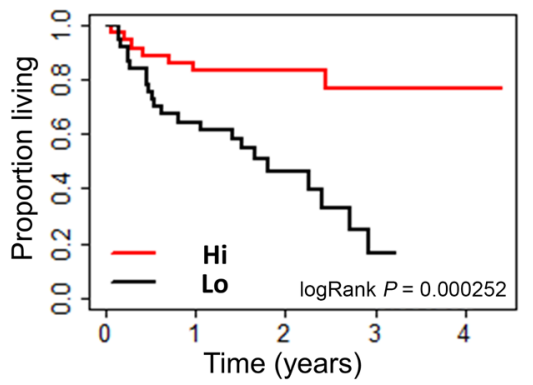

E CIBERSORT M1/M2 proportion ratio
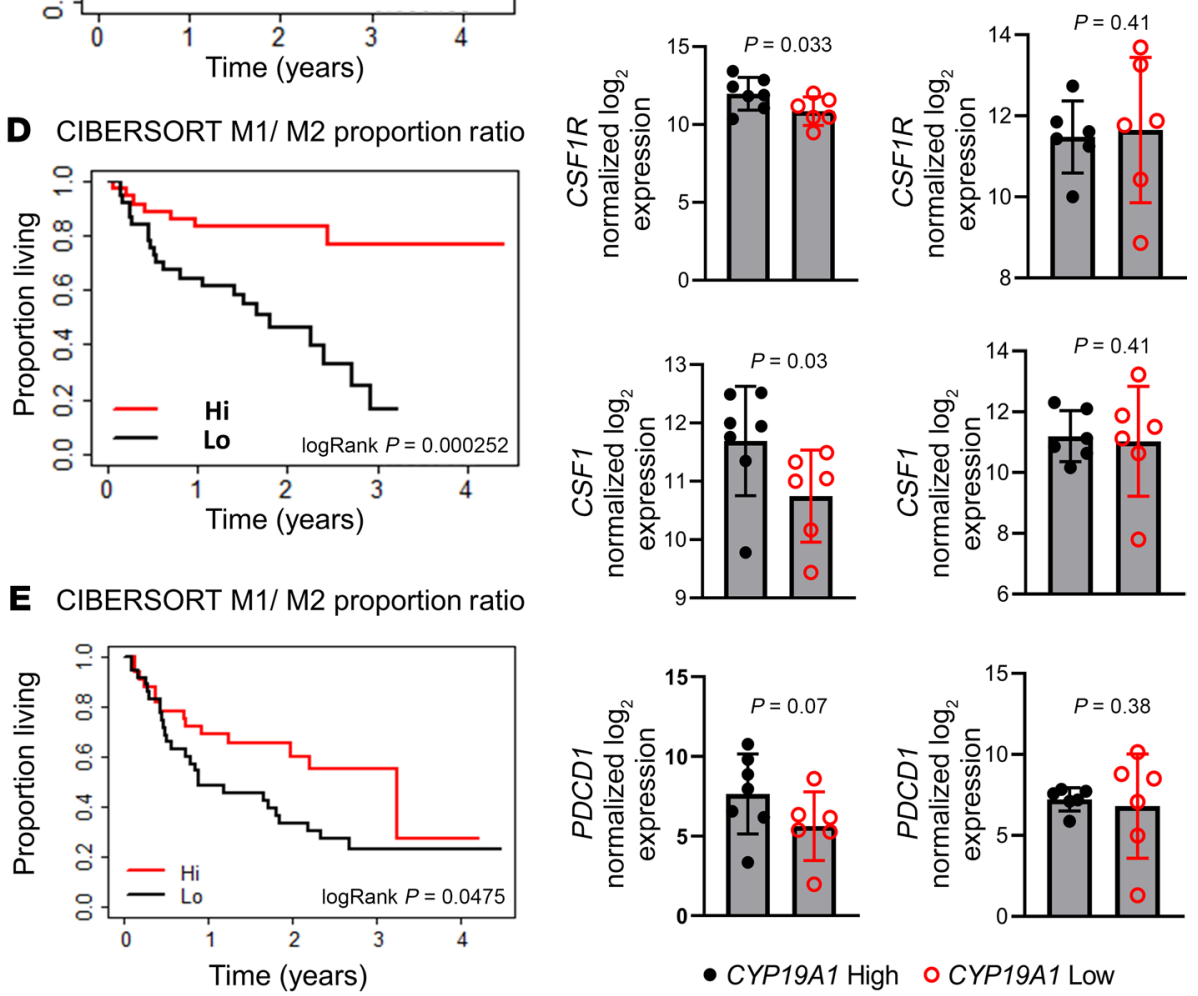

\begin{tabular}{|l|r|r|r|}
\hline & CR & PR & SD \\
\hline PR & $0.91 \mathrm{NA}$ & NA \\
\hline SD & 0.023 & 0.0016 & NA \\
\hline PD & 0.011 & 0.0051 & 0.24 \\
\hline \multicolumn{3}{l|}{ Pairwise $t$ test with BH $P$ value } \\
adjustment
\end{tabular}
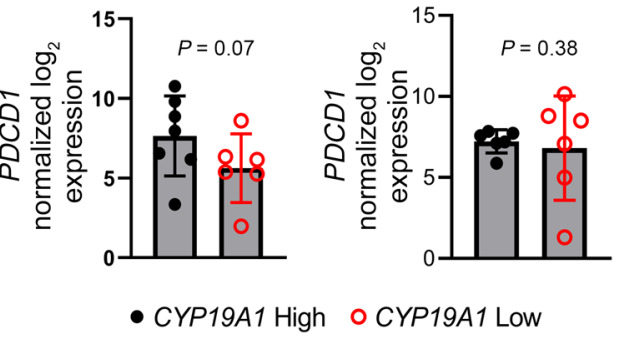

Figure 1. A decreased M1/M2 ratio compromises the immunotherapy benefit for patients with melanoma. (A and B) Relative proportion of M1 macrophages as determined by CIBERSORT or the ratio of M1/M2 macrophages in patients with melanoma, parsed by their response to immunotherapy in the same patient cohort. BH, Benjamini-Hochberg; Ipi, ipilimumab; Nivo, nivolumab; Pembro, pembrolizumab; RECIST, Response Evaluation Criteria in Solid Tumors. (C and D) Median overall survival for all patient cohorts (32) treated with immunotherapy with either high or low proportions of M1 macrophages or M1/M2 ratios as determined by CIBERSORT. (E) Median overall survival for all patient cohorts treated with ipilimumab alone (33) or either pembrolizumab or nivolumab alone (34), with either high or low M1/M2 signature ratios as determined by CIBERSORT. (F and G) CD68, CSF1, CSF1R, and PDCD1 expression in patients with melanoma who were classified as nonresponders $(n=13)$ or responders $(n=12)$ to $\alpha$-PD- 1 therapy, obtained from data sets from Hugo et al. (34). Both responders and nonresponders were stratified according to CYP19A $7^{\mathrm{hi}}$ and $C Y P 19 A 7^{\mathrm{lo}}$ median expression. Significance was calculated using a paired Student's $t$ test (A and $\mathbf{B})$, an unpaired Student's $t$ test (F and $\mathbf{G}$ ), and a log-rank test (C-E).

regarding the expression of nuclear ERs in melanoma cancer cells, the extent to which these receptors play a role in tumor progression remains to be determined (17). ERs have also been shown to be expressed in several different cell types within the tumor microenvironment (TME) and probably play a role in determining the tumor response to ER modulators. Indeed, 17ß-estradiol (E2) working through ER $\alpha$ expressed in endothelial cells in the TME has been shown to induce tumor growth by improving tumor angiogenesis and protecting tumor cells against hypoxia and necrosis (18). Further, ER actions have been studied in different immune cell types in different diseases (19-21), but the extent to which the ER influences immune cell biology within the TME has not been examined in detail. Recently, it has been demonstrated in ovarian cancer that $\mathrm{E} 2$ can create an immune-suppressive TME by promoting the mobilization of myeloid-derived suppressor cells (MDSCs) from bone that function to suppress tumor immunity and increase tumor growth (22). Although this study demonstrates that ER function is important for MDSC mobilization, the TME is infiltrated with multiple other myeloid cell types such as DCs, monocytes, and tumor-associated macrophages (TAMs), all of which affect tumor immunity (23). Notably, ERs have been shown to play a critical role in the development and functionality of these myeloid cell types $(24,25)$. However, the extent to which ER function regulates myeloid cell-T cell crosstalk within the TME and how it affects ICB responses are not known.

In this study, we explored how E2 modulates the immune cell function and repertoire within the melanoma TME and how this influences tumor growth in established murine models of 
A
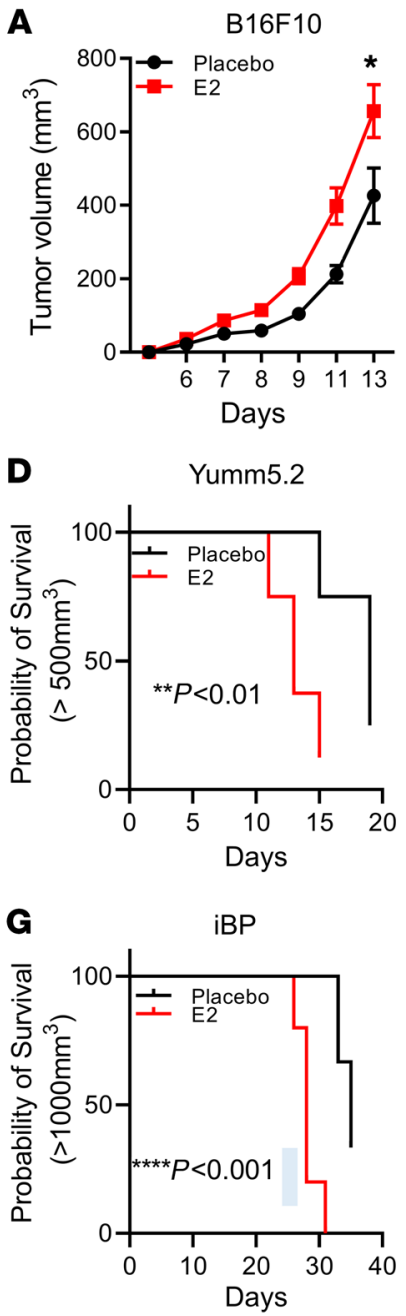

B
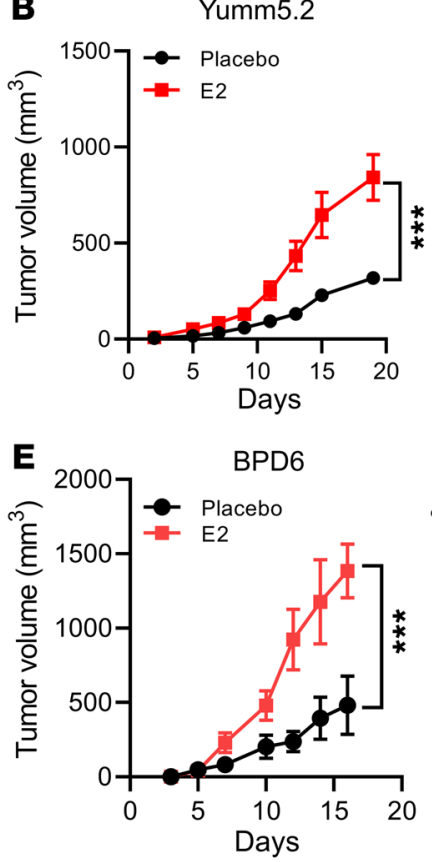

H

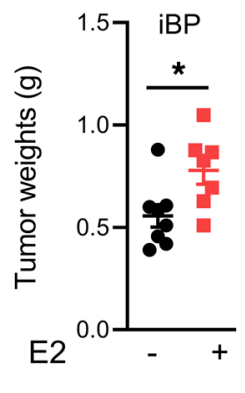

C
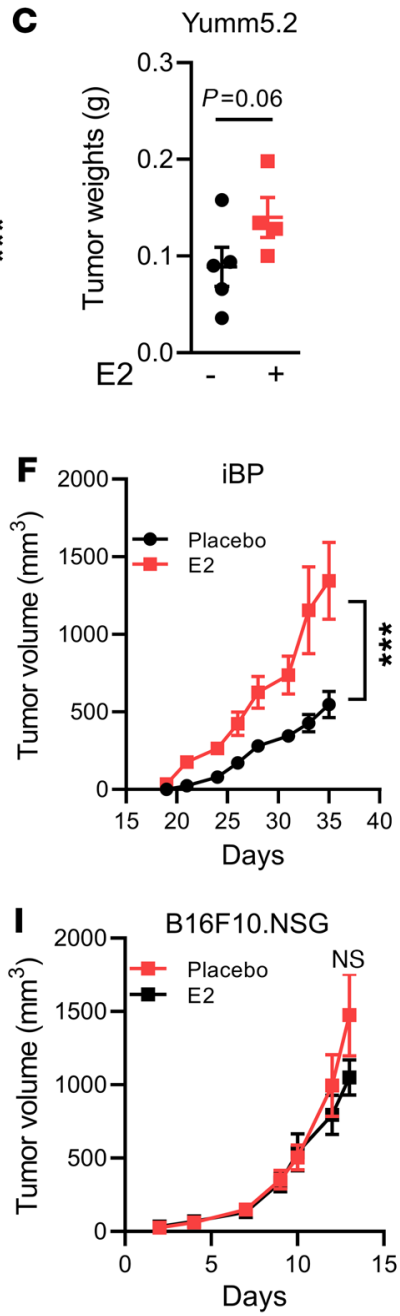

Figure 2. E2 promotes melanoma tumor growth. (A, B, and E) Subcutaneous tumor growth of B16F10 $\left(1 \times 10^{5}\right.$ cells) $(n$ $=10)$, Yumm5.2 (0.5 $\times 10^{5}$ cells) $(n=8)$, and BPD6 $\left(0.5 \times 10^{5}\right.$ cells $)(n=5)$ cells in syngeneic $[57 \mathrm{BL} / 6]$ ovariectomized hosts supplemented with placebo or E2. (C) Weights of YuMM5.2 tumors, from experiments in Figure 1B. (D) Survival of mice harboring YuMM5.2 tumors, from the experiment in Figure 1B. (F) Tumor growth in iBP female mice that were ovariectomized and supplemented with either placebo or E2 pellets $(n=5)$. Tumor formation in these mice was induced with a single intradermal dose of $150 \mu \mathrm{g}$ 4-hydroxytamoxifen (4OHT). (G and $\mathbf{H}$ ) Survival and tumor weights (placebo vs. E2), from experiments in Figure $1 F(n=6)$. (I) B16F10 $\left(1 \times 10^{5}\right.$ cells) tumor growth in ovariectomized NSG mice supplemented with placebo or E2 $(n=10)$. Results in $\mathbf{A}$, $\mathbf{B}, \mathbf{E}$, and $\mathbf{F}$ are representative of 2 independent experiments. Data are expressed as the mean \pm SEM. ${ }^{*} P<0.05,{ }^{* *} P<$ $0.01,{ }^{* *} P<0.001$, and ${ }^{* * * *} P<0.001$, by Student's $t$ test ( $\mathbf{C}$ and $\mathbf{H}$ ), log-rank test (D and $\mathbf{G}$ ), and 2-way ANOVA followed by Bonferroni's multiple-correction test (A, B, E, F, and I). this disease. Specifically, we have determined that a primary action of E2 is to facilitate the polarization of macrophages toward an immune-suppressive state in the TME, an action that is characterized by an enhanced ability to promote tumor growth and, in an indirect manner, suppress cytotoxic T cell responses. Further, we provide evidence that pharmacological inhibition of E2 signaling, using the selective estrogen receptor downregulator (SERD) and antagonist fulvestrant reversed E2-enhanced melanoma tumor growth by stimulating the establishment and maintenance of a proimmunogenic TME characterized by an increased presence of activated $\mathrm{CD}^{+} \mathrm{T}$ cells. Importantly, in preclinical models of melanoma, fulvestrant treatment increased the efficacy of $\alpha-\mathrm{PD}-1$ and $\alpha$-CTLA 4 treatment, providing the rationale for a clinical trial that will soon be initiated to evaluate the utility of combining contemporary SERDs with standard-of-care immunotherapies to maximize the therapeutic response in patients with melanoma.

\section{Results}

Decreased M1/M2 TAM ratio compromises the benefit of ICB therapy in patients with melanoma. Myeloid cell infiltration has been associated with poor outcomes in multiple cancer types (26-31). However, the extent to which tumor-infiltrating myeloid cells influence the response to immunotherapy in patients with melanoma has not been explored. To address this issue, we evaluated potential correlations between the number and characteristics of tumor-infiltrating myeloid cells and the patient's response to ICB using published transcriptomic data sets for patients with melanoma who had received standard-of-care ICB (32-34). The predominant suppressive myeloid cells in the TME are MDSCs and TAMs. To determine whether MDSCs play a role in predicting a patient response to ICB, we used validated MDSC gene signatures (35-39) to analyze transcriptomic data (32) on patients with melanoma who had received $\alpha-P D-1$ (nivolumab or pembrolizumab) or $\alpha$-CTLA4 (ipilimumab) therapy, either alone or in combination. As shown in Supplemental Figure 1, A-E (supplemental material available online with this article; https:// doi.org/10.1172/JCI151347DS1), MDSC signatures were not predictive of a patient's response to ICB or survival. In contrast, signatures from CIBERSORT (39) that determine the polarization state of TAMs are useful in predicting ICB response in the same data sets (32). Notably, enrichment of the M1 gene signature in tumors was associated with better responses (increased number of complete responders [CRs] and partial responders [PRs]) when compared with patients with stable disease (SD) or progressive disease (PD) (Figure 1A). We also observed a sim- 
A

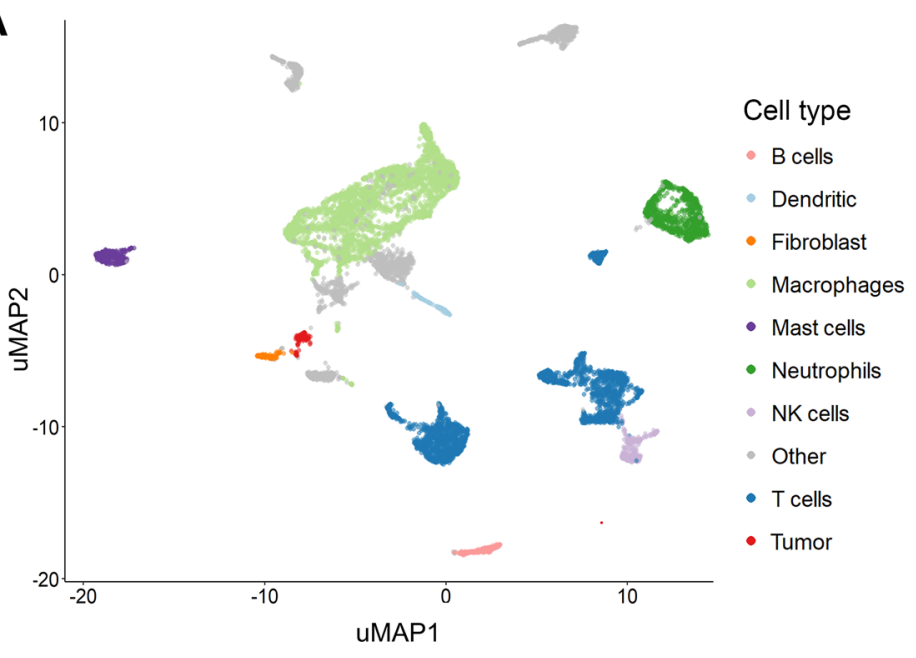

D
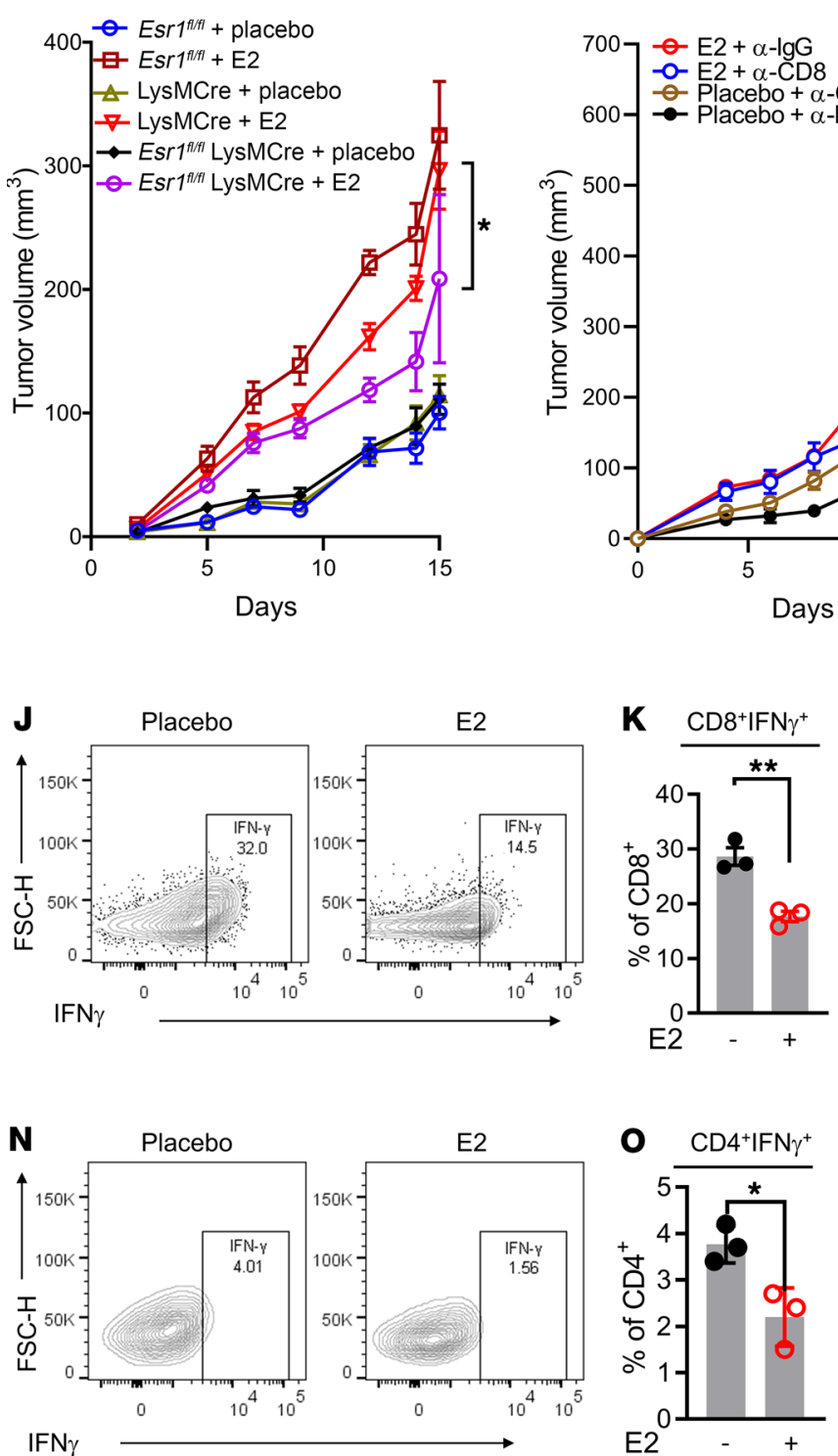

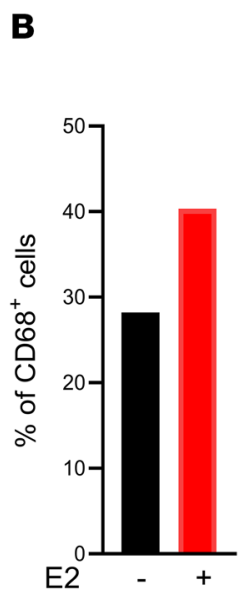

C $-E s r^{1 / n}+$ placebo

世Esr $1^{n+1}+\mathrm{E} 2$

$15007-$ LysMCre + placebo

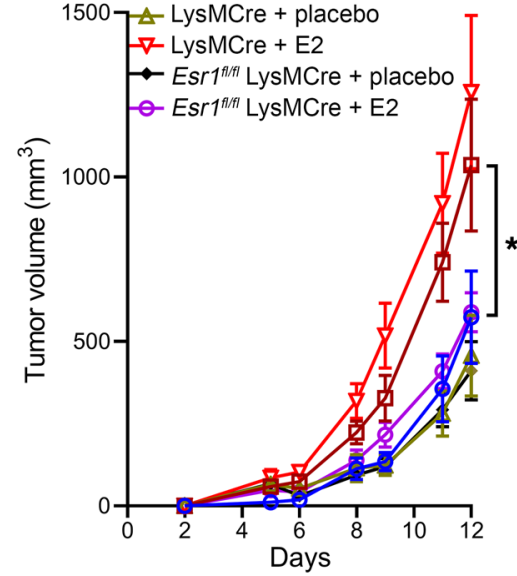

F $\quad \mathrm{CD}^{+} \mathrm{CFSE}^{+}$

G
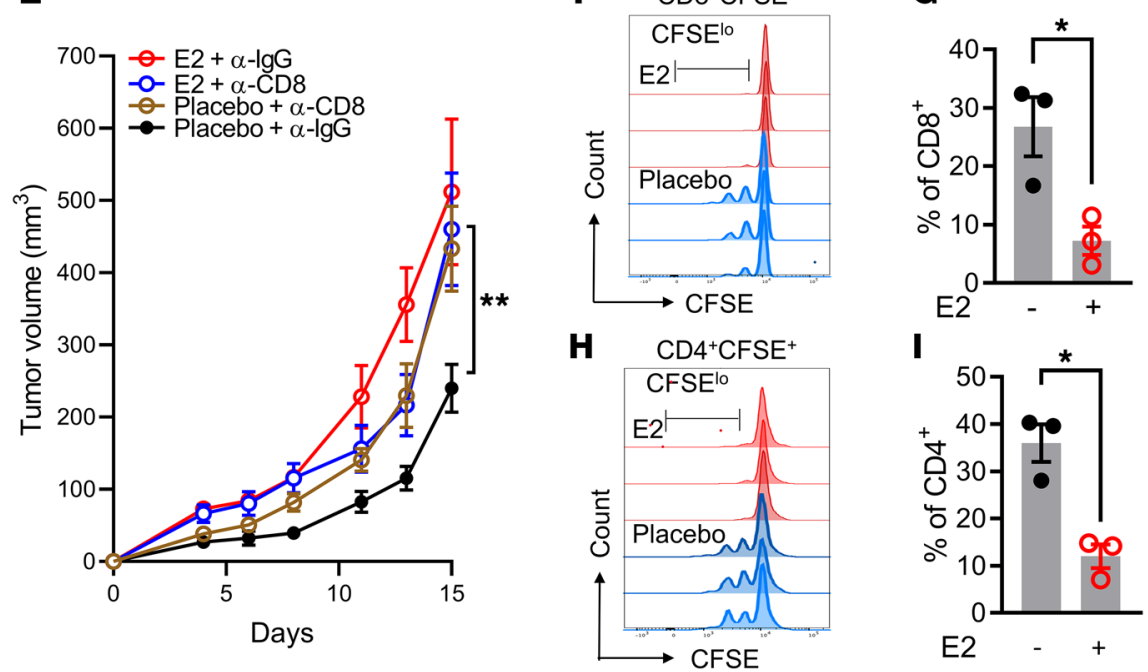
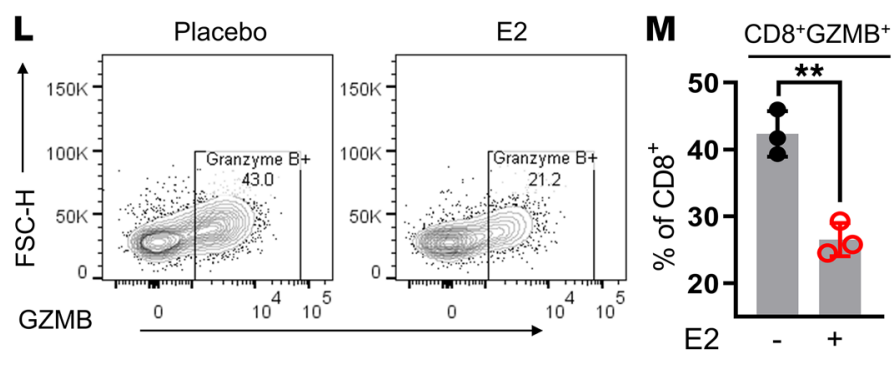
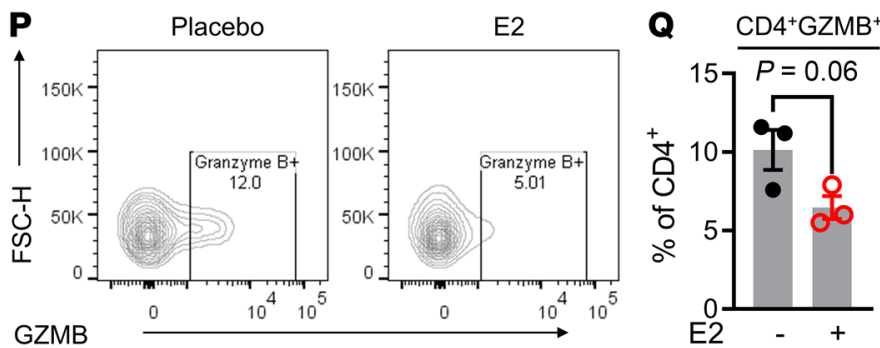
Figure 3. E2 regulates myeloid cell function in the TME. (A and B) UMAP plots of expression profiles for tumor-infiltrating immune cells (CD45) ( $n$ = 3 tumors/treatment, pooled together) isolated from iBP tumors. Each dot represents an individual cell (A). Percentage of $\mathrm{CD68} 8^{+}$macrophages/ monocytes among all sequenced cell types determined by scRNA-Seq in placebo- versus E2-treated samples (B). (C and D) Syngeneic tumor growth of B16F10 $\left(1 \times 10^{5}\right)$ and YuMM5.2 $\left(5 \times 10^{5}\right)$ cells in myeloid ER $\alpha$-knockout $\left(E s r 7^{f / f l}\right.$ LysMCre) and littermate control (Esr1 $7^{f / f l}$ and LysMCre) mice that were ovariectomized and supplemented with either placebo or E2 pellets. Esr fl/fl plus placebo (blue, $n=10$ ); Esr $7^{f / f l}$ plus E2 (maroon, $n=8$ ); LysMCre plus placebo (brown, $n=7$ ); LysMCre plus E2 (red, $n=7$ ); Esrr $7^{f l / f 1}$ LysMCre plus placebo (black, $n=8$ ); and Esr $7^{f / f f}$ LysMCre plus E2 (purple, $n=8$ ). (E) Tumor growth of YuMM5.2 cells $\left(5 \times 10^{5}\right)$ in CD8 ${ }^{+}$T cell-depleted C57BL6/] hosts that were ovariectomized and supplemented with placebo or E2 ( $n=$ 8 mice/treatment). (F-I) T cell proliferation was assessed after coculturing with tumor-infiltrating CD11 $\mathrm{b}^{+}$cells isolated from iBP mice treated with either placebo or E2. Shown are representative CFSE dilution plots of CD8 ${ }^{+}$ (F) and $\mathrm{CD}^{+}(\mathbf{H})$ cells. Quantification of CFSE low/negative $\mathrm{CD}^{+}(\mathbf{C})$ and $\mathrm{CD} 4^{+}(\mathrm{I})$ populations, expressed as a percentage of $\mathrm{CD} 8^{+}$and $\mathrm{CD} 4^{+} \mathrm{T}$ cells $(n=3)$. Results are representative of 2 independent experiments. (J-Q) Representative flow cytometric plots and the percentage of IFN- $\gamma^{+} \mathrm{CD} 8^{+}$

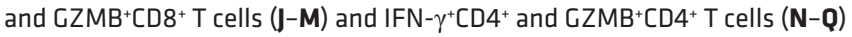
after 72 hours of coculturing with tumor-infiltrating CD11 b ${ }^{+}$myeloid cells isolated from iBP mice treated with either placebo or E2 ( $n=3 /$ group). Data indicate the mean \pm SEM. ${ }^{*} P<0.05$ and ${ }^{* *} P<0.01$, by Student's $t$ test (G, $\mathbf{I}, \mathbf{K}, \mathbf{M}, \mathbf{O}$, and $\mathbf{Q}$ ) and 2-way ANOVA followed by Bonferroni's multiple-correction test (C-E). FSC-H, forward scatter height.

ilar trend in patient prognosis when patients were parsed as a function of high versus low intratumoral M1/M2 macrophage ratios (Figure 1B). Enrichment of the M2 signature alone did not correlate with patient prognosis (Supplemental Figure 2A). Using the same data set (32), we also assessed whether the macrophage gene signature was associated with overall survival in patients with melanoma receiving immunotherapies. Similar to what we observed with patient prognosis (Figure 1, A and B), an enrichment of either the M1 gene signature or the M1/M2 ratio gene signature, but not enrichment of the M2 signature, was associated with better overall survival (Figures 1, C and D, and Supplemental Figure 2B). Interestingly, we also noted a positive association between the enrichment of an M1 gene signature, or the M1/M2 ratio gene signature, with patient prognosis and survival when the patients were parsed for those who received $\alpha-\mathrm{PD}-1$ monotherapy alone (Supplemental Figure 2, C-H), whereas those patients who received dual therapy showed a nonsignificant trend in this association (Supplemental Figure 2, $\mathrm{I}-\mathrm{N})$. Additionally, an increase in the intratumoral M1/M2 ratio predicted better survival in patients with melanoma in The Cancer Genome Atlas (TCGA) skin cutaneous melanoma (SKCM) data set (Supplemental Figure 3, A-C). The prognostic utility of assessing the intratumoral M1/M2 macrophage ratio was confirmed in independent data sets derived from melanoma patients treated with immunotherapy (Figure $1 \mathrm{E}$ and refs. 33, 34). It has been reported in several studies that sex influences the patient's response to immunotherapy in melanoma, with females receiving less benefit from ICB than males $(14,40)$. Motivated by these observations and previous studies demonstrating that female steroid hormone estrogens (E2) affect macrophage differentiation and polarization $(19,21)$, we hypothesized that estrogens may modulate the TME to promote immunotherapy resistance.
It was of significance, therefore, that we found that increased expression of CYP19A1, the enzyme that controls the rate-limiting step in estrogen biosynthesis, was correlated with increased TAM accumulation in patients with melanoma who were nonresponsive to ICB (Figure 1, F and G, and ref. 34). Importantly, stratification of patients on the basis of tumor expression of CYP19A1 mRNA revealed that its elevated expression was associated with expression of the macrophage markers CD68, CSF1, CSF1R, and the $\mathrm{T}$ cell exhaustion marker PDCD1 (Figure 1F) in nonresponders, whereas no such associations were identified in responder patient populations (Figure 1G). These results suggest that E2 may be causally involved in the establishment of an immune-suppressive TME through modulation of TAM biology, a hypothesis that we proceeded to test experimentally.

E2 promotes melanoma tumor growth. The results of studies addressing whether ERs are expressed within melanoma cells and tumors are equivocal. While some studies have demonstrated low expression of $E R \alpha$ and $E R \beta$ in human melanoma tumors by immunohistochemical staining $(41,42)$, the functionality of these receptors within tumor cells is unknown. Thus, we evaluated the expression of ER $\alpha$ in B16F10 and YuMM5.2 mouse melanoma cells following siRNA-mediated knockdown of Esr1. We used $E R \alpha^{+}$ MCF7 cells as a positive control for ER $\alpha$ expression. We detected weak ER $\alpha$ protein expression in YuMM5.2 cells, and this was depleted upon siRNA treatment (Supplemental Figure 4, A and B). By immunoblotting, we were unable to detect $\mathrm{ER} \alpha$ protein in B16F10 cells (a band migrating at approximately the same size as $\mathrm{ER} \alpha$ was not depleted upon siRNA treatment, despite a marked reduction of Esr1 mRNA, expressed at very low levels). Regardless, treatment of either cell with E2 did not lead to changes in the expression of classical ER target genes (Pgr and Cxcl12) (Supplemental Figure 4C), nor did it induce proliferation (Supplemental Figure 4, D and E). Collectively, these data validate the use of these cell models to study the cancer cell-extrinsic actions of estrogens and ER modulators on the pathobiology of melanoma. To this end, we subcutaneously injected B16F10, YuMM5.2, or BPD6 melanoma cells into ovariectomized syngeneic mice supplemented with either placebo or E2 pellets $(0.01 \mathrm{mg} / 60$ days continuous release). As expected, E2 administration resulted in an increase in uterine wet weights in the ovariectomized mice (Supplemental Figure 4F). As shown in Figure 2, A-E, treatment with E2 significantly increased tumor growth in all 3 syngeneic models compared with the placebo-treated control mice. To further validate our observations in a more clinically relevant system, we used an autochthonous mouse model in which tumor growth was driven by concomitant conditional activation of $\mathrm{B}-\mathrm{Raf}^{\mathrm{V}} \mathrm{V}^{\mathrm{V}} \mathrm{E}$ and homozygous deletion of Pten in melanocytes (Braf ${ }^{\mathrm{m} 1 \mathrm{Mmcm}}$ Pten $^{f / f l}$ mTyr-CreERT2, hereafter referred as iBP; ref. 43). This mouse model faithfully resembles human melanomas harboring $B R A F$ and PTEN mutations. Similar to the syngeneic models, administration of E2 in ovariectomized mice accelerated tumor growth in the iBP model compared with their placebo-treated counterparts (Figure 2, F-H). The slower tumor growth kinetics that were imparted by ovariectomy disappeared when B16F10 cell-derived tumors were grown in NOD.Cg-Prkdc $c^{\text {cid }} \mathrm{Il} 2 \mathrm{rg}^{\mathrm{tm} l \mathrm{Wjl}} / \mathrm{SzJ}$ (NSG) mice (Figure 2I), suggesting that the actions of E2 on tumor growth were likely mediated by an immune cell or cells. 

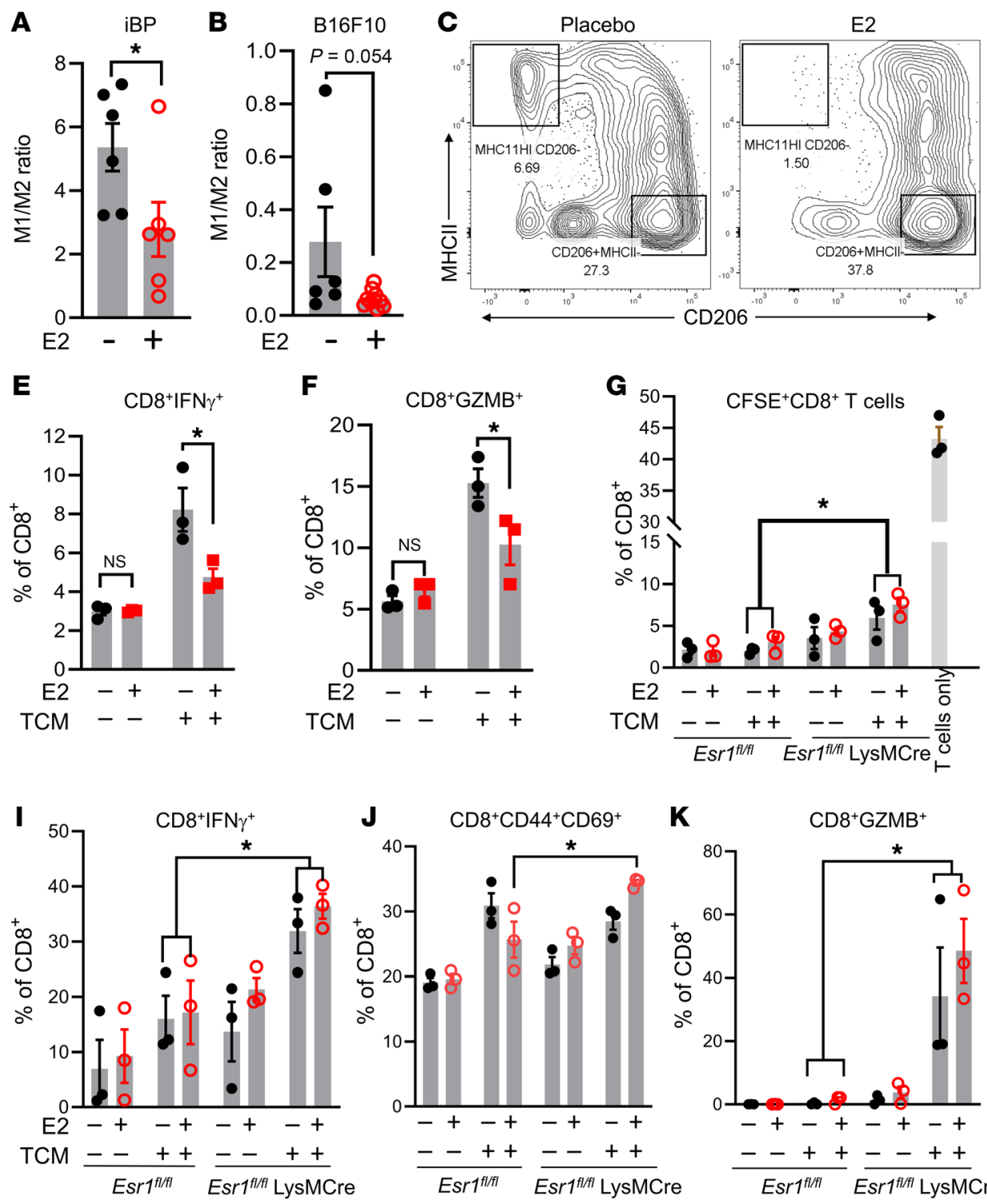

K $\mathrm{CD}^{+} \mathrm{GZMB}^{+}$

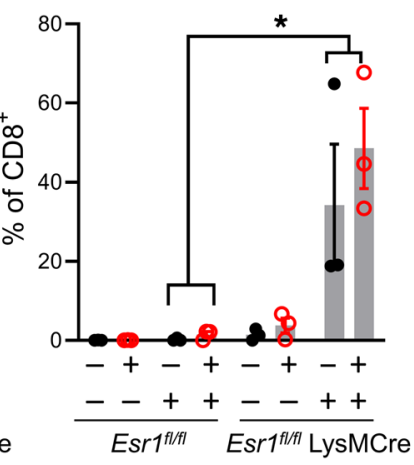

$\mathbf{L}$

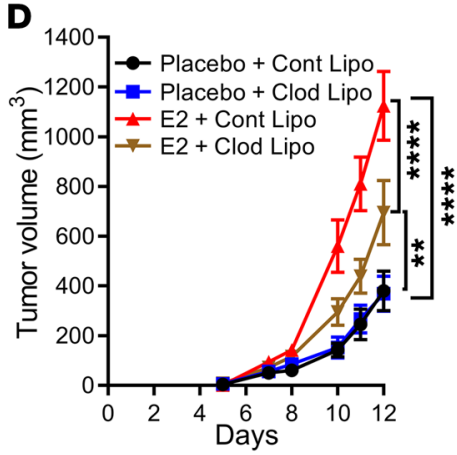

H
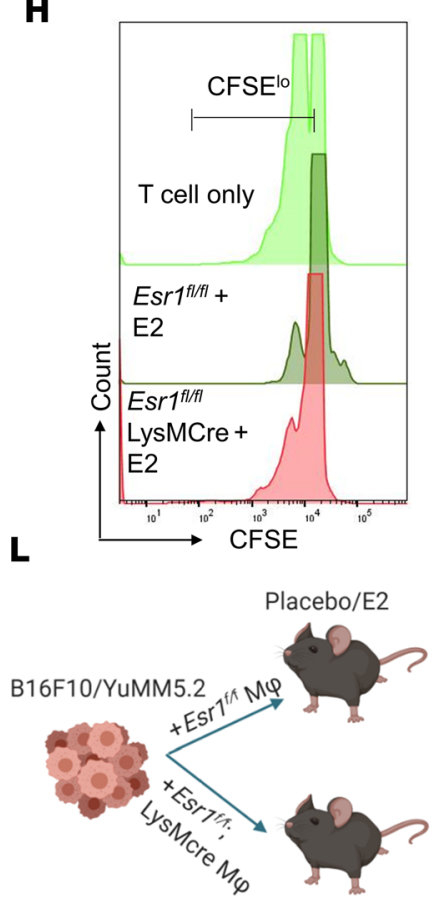

M

$\mathbf{N}$

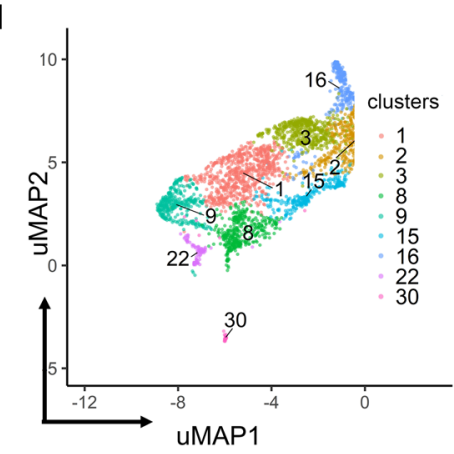

- cell_color
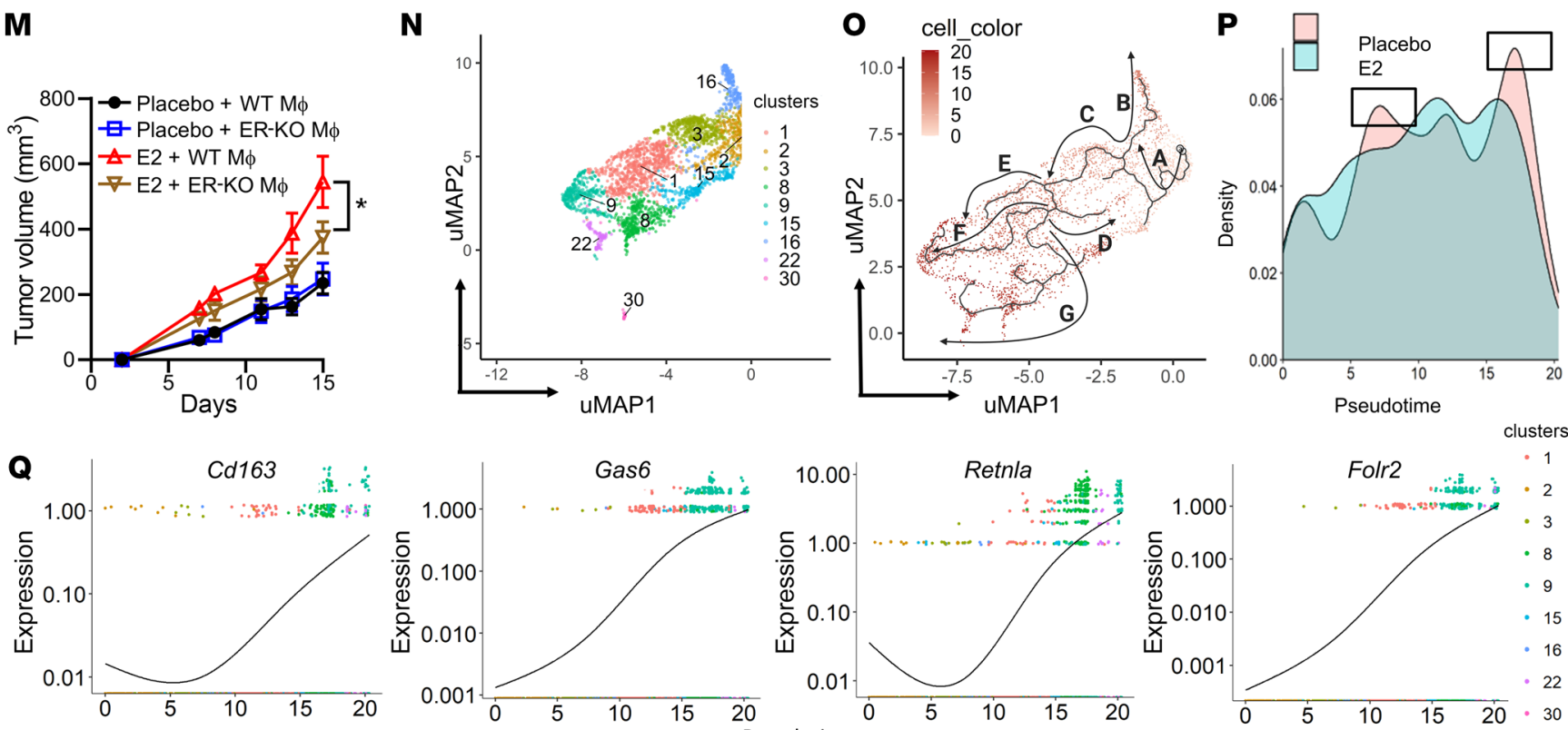
Figure 4. E2 regulates TAM function. (A-C) Ratio of $M 1 / M 2$ macrophages in $\mathrm{BBP}(n=6)(\mathbf{A})$ and B16F10 $(n=6-10)$ (B) tumors from placebo- and E2-treated mice and representative flow cytometric plots of $M 2$ and M1 macrophages in the B16F10 model (C). (D) Growth of B16F10 tumors ( $n=$ 12) upon depletion of macrophages by clodronate liposomes (Clod Lipo) in ovariectomized mice supplemented with placebo or E2. Cont Lipo, control liposome. (E and $\mathbf{F}$ ) Quantification of IFN- $\gamma^{+} \mathrm{CD} 8^{+} \mathrm{T}$ cells $(\mathbf{E})$ and $\mathrm{CZMB} \mathrm{B}^{+} \mathrm{CD} 8^{+}$ T cells $(\mathbf{F})(n=3)$ that were cocultured with BMDMs differentiated in NM or TCM and treated with either DMSO or E2 $(1 \mathrm{nM})$. (G and $\mathbf{H})$ CFSE dilution and quantification representing the proliferation of CFSE ${ }^{10 /-}$-CD8 ${ }^{+} T$ cells ( $n$

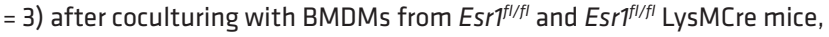
differentiated in either normal media or TCM (B16F10), followed by treatment with either DMSO or E2 (1 nM). (I-K) Quantification of CD8 $8^{+} \mathrm{FN}-\gamma^{+}$, $\mathrm{CD}^{+}{ }^{+} \mathrm{CD} 44^{+} \mathrm{CD}^{+} 9^{+}$, and $\mathrm{CD} 8{ }^{+} \mathrm{CZMB}^{+} \mathrm{T}$ cells $(n=3)$ from the same experiment as in G. (L) Schematic of tumor comixing methodology. (M) Syngeneic tumor growth of YuMM5.2 $\left(5 \times 10^{5}\right)$ cells comixed with BMDMs from either

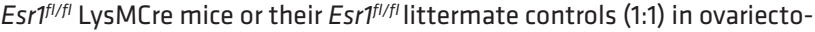
mized mice supplemented with either placebo or E2. Esr ffl/fl BMDMs plus

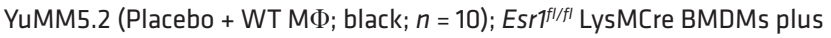

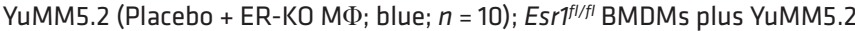

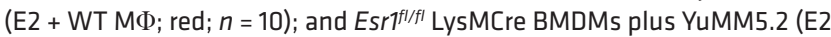
+ ER-KO MФ; brown; $n=10$ ). (N) uMAP representation of macrophage/ monocyte subclusters as determined by scRNA-Seq. (0) Trajectory analysis depicting the differentiation of monocytes into different lineages of macrophages. (P) Density of cells in macrophage/monocyte subclusters along a pseudotime gradient. (Q) Expression of M2-associated genes ( $C \mathrm{C} 163$, Lgr2, Retnla, and Folr2) in macrophage clusters along the pseudotime axis. Data in $\mathbf{E}-\mathbf{K}$ are representative of 2 independent experiments. Data are expressed as individual data points and indicate the mean \pm SEM. ${ }^{*} P<$ 0.05 , ${ }^{*} P<0.01$, and ${ }^{* * *} P<0.0001$, by Student's $t$ test (A and B), 1-way ANOVA (E-G, I-K) and by 2-way ANOVA followed by Bonferroni's multiple-correction test ( $\mathbf{D}$ and $\mathbf{M}$ ). $M \Phi$, macrophages.

E2 regulates the function of tumor-associated myeloid cells. To determine how E2 treatment affects the tumor immune microenvironment, we performed single cell RNA-Seq (scRNA-Seq) analysis of tumor-infiltrating immune cells isolated from iBP tumors treated with either placebo or E2. Unsupervised clustering analysis using uniform manifold approximation and projection (uMAP) revealed global differences in tumor-infiltrating immune cells when comparing placebo and E2 treatments and identified clusters of immune cells that had unique transcriptional profiles. Comparison of cell type signature(s) with the Immunological Genome (ImmGen) database and known cell type markers (Supplemental File 1) resulted in the identification of 9 macrophage/myeloid clusters, 10 lymphoid clusters, 2 neutrophil clusters, 2 DC clusters, and $1 \mathrm{~B}$ cell, NK cell, and mast cell cluster (Figure 3A and Supplemental Figure 5A). Analysis of the scRN-Seq data set also revealed that the majority of Esr1 transcripts were expressed in cells within the myeloid lineage, whereas the expression of Esr2 and Gper was minimal to undetectable (Supplemental Figure 5, B-D). Differences in the immune cell repertoires from placebo- and E2-treated tumors were also evident (Supplemental Figure 6A). Notably, E2 treatment led to the expansion of and marked changes in gene expression in the CD68 ${ }^{+}$monocyte/TAM clusters (Figure 3B and Supplemental Figure 6B). To determine the functionality of ER signaling in the monocyte/TAM cluster, we genetically depleted $\mathrm{ER} \alpha$ in myeloid cells using a lysozyme-driven Cre-recombinase (Esr $1^{f / f l}$ LysMCre) to establish its role(s) in tumor responses to $\mathrm{E} 2$. ER $\alpha$ depletion in the myeloid lineage was confirmed in bone marrow-derived macrophages (BMDMs) isolated from Esr $1^{f / / f l}$ LysMCre and littermate Esr $1^{f l / f l}$ control mice (Supplemental Figure 6C). Subsequently, we used 8-week-old Esr $1^{f / f l}$ LysMCre and littermate control (Esr1 ${ }^{f l / f l}$ and LysMCre) mice to evaluate syngeneic tumor growth in the B16F10 and Yumm5.2 models, in the presence or absence of E2. The growth of B16F10 and YuMM5.2 tumors increased in response to $\mathrm{E} 2$ in $E s r f^{f / f l}$ and LysMCre mice, but this was not evident in Esr $1^{f / f l}$ LysMCre mice (Figure 3, C and D, and Supplemental Figure 6D). Analysis by flow cytometry of tumor-infiltrating immune cells revealed a decrease in M1 (proinflammatory macrophages) in E2-treated $E s r 1^{f / f l}$, but not Esr $1^{f / f l}$ LysMCre, animals (Supplemental Figure 6E). Myeloid cells can often manifest their actions by modulating other cell types in the TME either by facilitating the release of cytokines and/or by blunting antigen presentation to the adaptive immune cells. To understand whether T cells play a functional role in E2-induced tumor growth, we depleted CD $8^{+} \mathrm{T}$ cells with an $\alpha$-CD 8 antibody in mice engrafted with YuMM5.2 tumor cells in the presence or absence of E2. The efficacy of the $\mathrm{CD}^{+} \mathrm{T}$ cell depletion was confirmed by flow cytometric analysis (Supplemental Figure 6, F and $\mathrm{G})$. Antibody-mediated acute depletion of $\mathrm{CD} 8^{+} \mathrm{T}$ cells reversed the protective effects of ovariectomy on YuMM5.2 tumor growth but did not accelerate tumor growth in E2-treated mice (Figure $3 \mathrm{E})$. These results indicate the functional involvement of $\mathrm{CD}^{+} \mathrm{T}$ cells in E2-mediated tumor growth.

To define the extent to which E2-treated myeloid cells affect $\mathrm{T}$ cell functionality, we isolated $\mathrm{CD} 11 \mathrm{~b}^{+}$myeloid cells from iBP tumors treated with either placebo or E2. These cells were then coincubated with $\mathrm{CD}^{+} \mathrm{T}$ cells isolated from the spleens of non-tumor-bearing Pmel mice [Thy $1^{a} / \mathrm{Cy} \mathrm{Tg}(\mathrm{TcraTcrb}) 8 \mathrm{Rest} / \mathrm{J}$ ] for 72 hours. iBP tumors express gp100 (Pmel; ref. 44) that can be processed and presented by professional antigen-presenting cells to $\mathrm{T}$ cells that are specific to the antigen (gp100). Prior to coincubation, T cells were stained with CFSE dye and activated in the presence of suboptimal CD3/CD28. As assessed by CFSE dye dilution, it was apparent that $\mathrm{T}$ cell (both $\mathrm{CD} 4^{+}$and $\mathrm{CD} 8^{+}$) proliferation was significantly inhibited by coincubation with myeloid cells isolated from tumors from E2-treated mice compared with T cells that were incubated with myeloid cells isolated from placebo-treated mice (Figure 3, F-I). Additionally, myeloid cells from E2-treated mice also affected the cytotoxic capability of both $\mathrm{CD} 8^{+}$and $\mathrm{CD} 4^{+} \mathrm{T}$ cells, as demonstrated by decreased expression of IFN- $\gamma$ (Figure 3, J, K, N, and $\mathrm{O}$ ) and granzyme B (GZMB) (Figure 3, L, M, P, and Q). Taken together, these observations suggest that the ER $\alpha / \mathrm{E} 2$ axis increases the immunosuppressive activities of tumor-infiltrating myeloid cells. In this experiment, we did not define the phenotypic characteristics of the isolated myeloid cells, i.e., BMDMs versus resident macrophages. However, in subsequent experiments (see below), we determined that the suppressive effects of E2 were likely mediated by macrophages that differentiated from monocytes recruited to the tumor from the bone marrow.

E2 promotes the accumulation of immune-suppressive TAMs within the TME. We performed flow cytometry to characterize the myeloid cells within tumors isolated from iBP mice and from mice engrafted with syngeneic tumors (B16F10), treated with either placebo or E2 (Supplemental Figure 7A). We found that, quantitatively, the infiltration of immune cells $\left(\mathrm{CD} 45^{+}\right)$was similar in the 
A
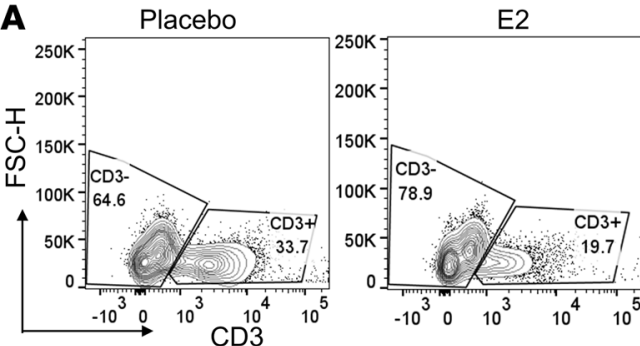

$\mathbf{E}$
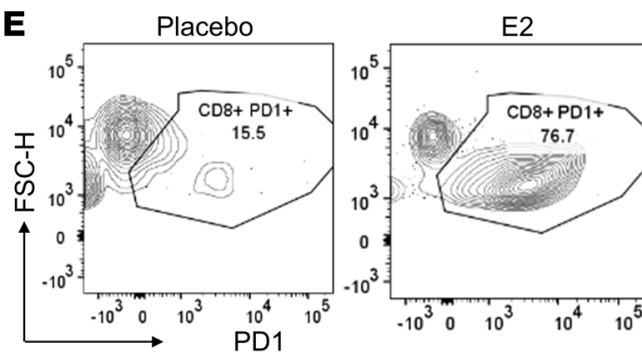

I

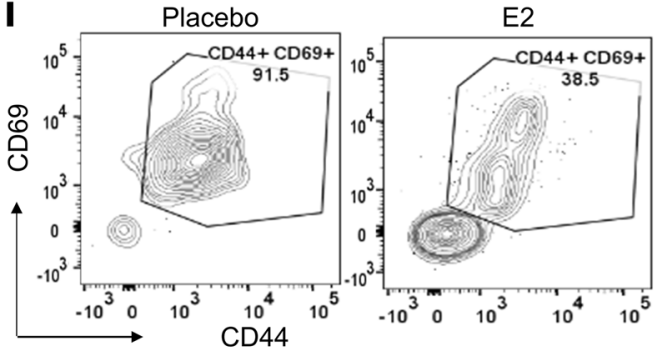

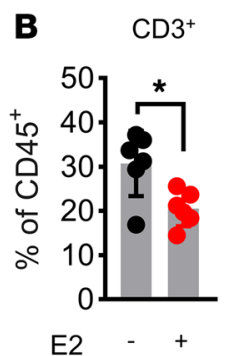
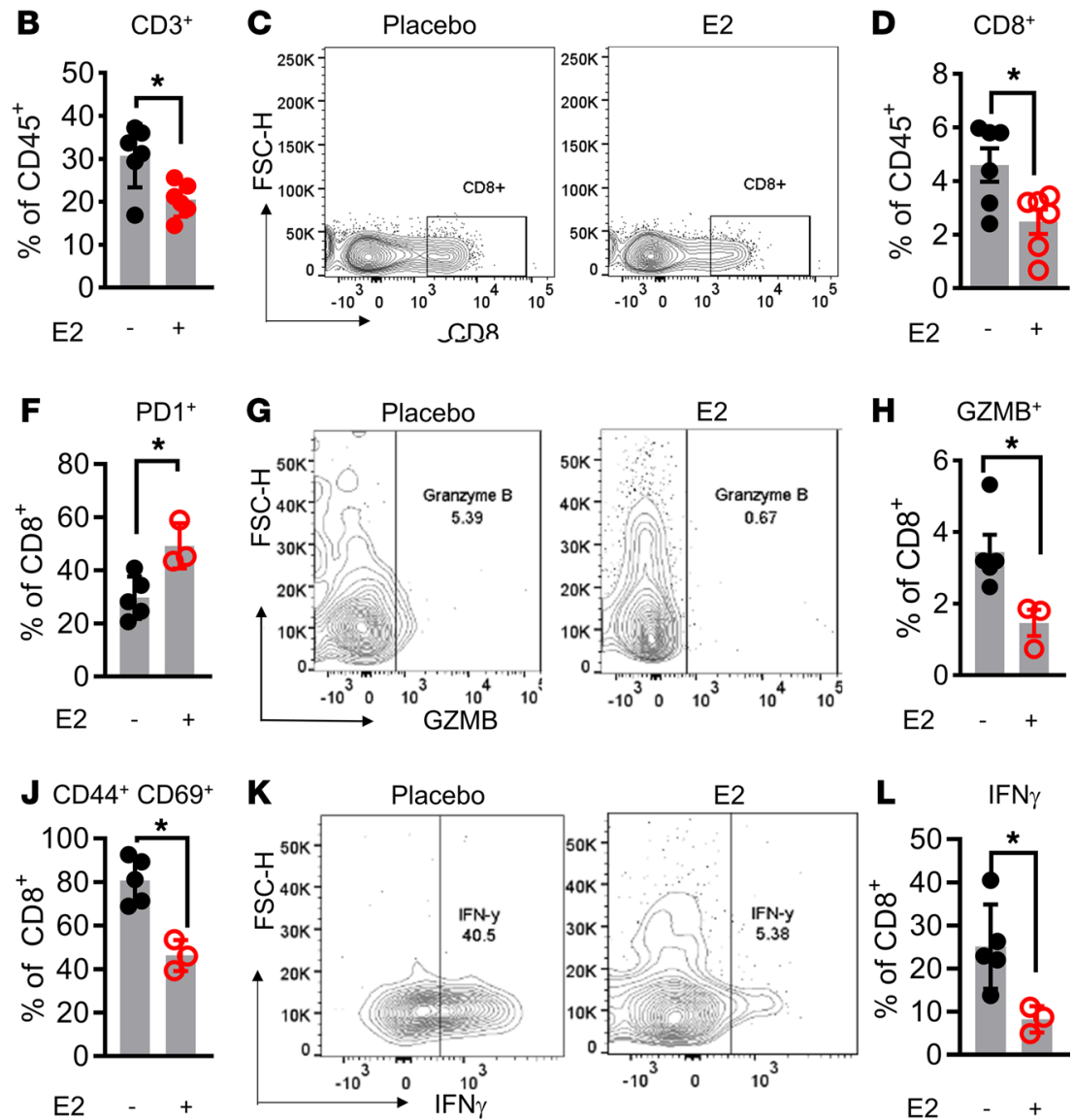

Figure 5. E2 suppresses antitumor $\mathbf{T}$ cell responses. Representative flow cytometric plots and quantification of $\mathrm{CD}^{+}(\mathbf{A}$ and $\mathbf{B})$ and $\mathrm{CD} 8^{+}(\mathbf{C}$ and $\mathbf{D}) \mathrm{TILs}$ in iBP tumors $(n=5-6)$ isolated from mice treated with either placebo (black) or E2 (red). (E-L) Representative flow cytometric plots and quantification of CD8 ${ }^{+}$PD-1 $1^{+}$ (E and $\mathbf{F}), \mathrm{CD}^{+} \mathrm{CZMB}^{+}(\mathbf{G}$ and $\mathbf{H}), \mathrm{CD}^{+} \mathrm{CD} 44^{+} \mathrm{CD69}^{+}(\mathbf{I}$ and $\mathbf{J})$, and $\mathrm{CD8} 8^{+} \mathrm{IFN}-\gamma^{+}(\mathbf{K}$ and $\mathbf{L}) \mathrm{T}$ cells in YuMM5.2 tumors from mice treated with placebo (black) or E2 (red) $(n=3-5)(\mathbf{H})$. Data are expressed as individual data points and indicate the mean \pm SEM. ${ }^{*} P<0.05$, by Student's $t$ test.

2 models and not affected by the treatment (Supplemental Figure 7, B and C). Qualitative assessments, however, revealed that E2 treatment decreased the ratio of intratumoral immunostimulatory $\mathrm{M} 1$ ( $\mathrm{MHCII}^{\mathrm{hi}} \mathrm{CD} 2 \mathrm{O6}^{-}$) macrophages to immunosuppressive M2 $\left(\mathrm{MHCII}^{\mathrm{lo}} \mathrm{CD}^{206^{+/ h i}}\right.$ ) macrophages (Figure 4, A-C). Of note, we did not observe any changes in the percentage of $\mathrm{Ly}^{6} \mathrm{C}^{+} / \mathrm{Ly} 6 \mathrm{G}^{+}$ MDSCs in tumors between the 2 treatment conditions (Supplemental Figure 7D). Depletion of macrophages using clodronate liposomes decreased melanoma tumor growth in E2-treated mice but was without any effect in the placebo-treated mice (Figure $4 \mathrm{D}$ and Supplemental Figure 7E). To demonstrate a direct effect of E2 on macrophage polarization (and function), bone marrow progenitor cells were differentiated into macrophages in the presence of macrophage-CSF (M-CSF) and either normal media or $30 \%$ tumor-conditioned media (TCM) from B16F10 cells. The addition of TCM allowed us to partially mimic the TME, where tumor-derived factors influence the differentiation and polarization of macrophages (45). Following differentiation, we treated the macrophages acutely with either DMSO or E2 $(1 \mathrm{nM})$ and then polarized them to an M2 state by the addition of IL-4. We subsequently cocultured the polarized macrophages with suboptimally activated T cells (CD3/CD28 and IL-2) isolated from spleens of non-tumor-bearing mice for 72 hours, following which they were treated with protein transport inhibitors (monensin and brefeldin) for 6 hours to prevent the release of cytokines and chemokines. Flow cytometric analysis revealed that $\mathrm{T}$ cells that were coincubated with either placebo- or E2-treated (1 nM) macrophages in normal media (NM) showed no change in the expression of IFN- $\gamma$ or GZMB. The basal expression of GZMB and IFN- $\gamma$ in T cells was significantly increased upon exposure to macrophages cultured in TCM. However, when T cells were coincubated with E2-treated $(1 \mathrm{nM})$ macrophages differentiated in TCM, they show decreased expression of GZMB and IFN- $\gamma$ compared with expression in $\mathrm{T}$ cells that were coincubated with DMSO-treated macrophages (Figure 4, E and F). These results indicate that E2 treatment induce an immune-suppressive phenotype in tumor-conditioned macrophages, which in turn suppressed the cytotoxic capabilities of T cells. However, in the absence of TCM, macrophages did not affect $\mathrm{T}$ cell activity, even in the presence of $\mathrm{E} 2$.

To further explore the roles of ER $\alpha$ in macrophage polarization, we isolated and differentiated bone marrow progenitor cells from Esr $1^{f / f l}$ and Esr $1^{f / f l}$ LysMCre animals into BMDMs in NM or $30 \%$ TCM (B16F10). We treated the differentiated BMDMs from both $E s r 1^{f / f f l}$ and $E s r 1^{f / f l}$ LysMCre genotypes with either DMSO or E2 and then polarized them to M2 macrophages by the addition of IL-4 (24 hours). These macrophages were then coincubated for 72 hours 
with CFSE-stained and suboptimally activated $\mathrm{T}$ cells isolated from non-tumor-bearing mouse spleens. Quantification of CFSE dilution revealed a marked attenuation of $\mathrm{T}$ cell proliferation after incubation with BMDMs compared with T cells alone. We observed no difference in the proliferation of $\mathrm{T}$ cells when $\mathrm{T}$ cells were coincubated with macrophages differentiated in NM, regardless of the genotypes of the BMDMs and treatments. However, using BMDMs differentiated in TCM, we observed a significant increase in the proliferation $\left(\mathrm{CFSE}^{\mathrm{lo} /-}\right)$, activation $\left(\mathrm{CD} 44^{+} \mathrm{CD} 69^{+}\right)$, and cytotoxicity $\left(\mathrm{IFN}-\gamma^{+}\right.$and $\mathrm{GZMB}^{+}$) of T cells when they were incubated with BMDMs derived from Esr $1^{f / f l}$ LysMCre mice compared with those from $E s r 1^{f l / f l}$ mice, irrespective of the presence or absence of E2 (Figure 4, G-K). These results demonstrate that depletion of ER $\alpha$ in the macrophages enhanced their capacity to promote the proliferation of cytotoxic T cells $\left(\mathrm{GZMB}^{+}\right.$and IFN- $\left.\gamma^{+}\right)$. However, in contrast to previous experiments in which we observed a decrease in GZMB and IFN- $\gamma$ expression in T cells upon coincubation with E2-treated macrophages, the $\mathrm{T}$ cells in this experiment did not show a similar decrease in the expression of these cytotoxic $\mathrm{T}$ cell markers when coincubated with E2-treated ER $\alpha^{f / f l}$ macrophages (Figure 4, E and F vs. I and K). This result may have been due to differences in the underlying genetics (Esr $f^{f / f l}$ vs. WT). The importance of $\mathrm{ER} \alpha$ signaling in macrophages in modulating melanoma tumor growth was further probed in vivo by coinjecting YuMM5.2 or B16F10 tumor cells together with BMDMs (Supplemental Figure 8A) from either $E s r 1^{f / f l}$ or $E s r 1^{f / f l}$ LysMCre mice (1:1) (Figure $4 \mathrm{~L}$ ) into syngeneic ovariectomized C57BL/6J mice treated with placebo or E2. The tumor-promoting effects of E2 were significantly compromised when tumors (YuMM5.2 and B16F10) were implanted with BMDMs from Esr $1^{f / f l}$ LysMCre animals compared with BMDMs from $E s r 1^{f / f l}$ animals (Figure $4 \mathrm{M}$ and Supplemental Figure 8B). Taken together, these results indicate that the E2/ER $\alpha$ signaling axis in macrophages cooperates with tumor-derived factors to promote the establishment of an immune-suppressive TME that facilitates melanoma tumor growth.

Examination of the scRNA-Seq profiles revealed that the $\mathrm{CD}^{+} 8^{+}$monocyte/TAM population from E2-treated tumors expressed markers that were previously reported to be selectively upregulated in TAMs versus macrophages isolated from the lungs of non-tumor-bearing mice (Trem2, Apoe, Thbs1, Spp1) (Supplemental Figure 8C and ref. 46). Genes associated with inflammation and those encoding select chemokines (Itm2b, C1q) and M2 macrophage markers (Tspo, Vegfa, Tgm2) were also upregulated in the $\mathrm{CD} 68^{+}$cells from the E2-treated group (Supplemental Figure $8 \mathrm{C}$ and ref. 46). The $\mathrm{CD}^{+} 8^{+}$population was composed of cells from 9 different clusters (clusters 1, 2, 3, 8, 9, 15, 16, 22, and 30; Figure $4 \mathrm{~N}$ ). Pseudotime analysis of the developmental trajectories of the macrophage and monocyte populations (Supplemental Figure 8D) revealed several major branches representing different clusters of cells emerging from monocytes (Figure 4O). Among these cell populations, clusters 2, 3, and 16 expressed the monocytic markers Cd14 (Supplemental Figure 9A) and $L y 6 c 2$, with cluster 2 (arrow A, Figure 4O) showing higher expression of $L y 6 c 2$ (Figure $4 \mathrm{O}$ and Supplemental Figure 9B). The cluster 2 (arrow A, Figure 4O) population then bifurcated into 2 branches, cluster 3 (arrow C, Figure 4O) and cluster 16 (arrow B, Figure 4O), both of which expressed intermediate levels of $C \times 3 \mathrm{cr} 1$ (Supplemen- tal Figure 9C), but cluster 3 had higher expression of Ccr2 (Supplemental Figure 9D) compared with cluster 16. Thus, cluster 3 likely represented inflammatory monocytes, whereas cluster 16 cell populations were more similar to patrolling tissue-resident monocytes (47). Of note, both clusters 3 and 16 were increased in E2-treated tumors compared with tumors from placebo-treated mice (pseudotime block 5-10, boxed region, Figure 4P), while the percentage of $L y 6 C^{\text {hi }}$ monocytes (cluster 2 ) remained the same between the 2 treatments (Figure $4 \mathrm{P}$ and Supplemental Figure 9B). Cluster 3 further proceeded to a major branching point, leading to the formation of 4 different trajectories, mainly cluster 15 (arrow D), cluster 1 (arrow E), cluster 9 (arrow F), and clusters 8, 22, and 30 (arrow G, Figure 4O). Among these clusters, 1, 8, 22, 30 , and 15 all expressed genes associated with the MHC II complex (H2-Aa, H2-Ab, H2-Dmb1, and H2-Eb1) (Supplemental Figure 9, E-H). Clusters 1 and 15 additionally expressed the inflammatory gene $I l 1 b$ (Supplemental Figure 9I) and were likely composed of inflammatory or "M1-like" TAMs. Although cluster 1 remained unchanged, cluster 15 decreased upon E2 treatment (Supplemental Figure 9Q). Clusters 8, 22, and 30 expressed inflammatory genes (Cd72 and Tlr2; Supplemental Figure 9, J-K) in addition to genes of the MHC II complex, however they also expressed genes associated with M2 macrophages (Mrc1; Supplemental Figure 9L). While the exact functionality of these macrophage subsets is not clear, phenotypically they were analogous to the population of circulating cells of monocyte/macrophage lineage that express markers of both M1 and M2 cell phenotypes as reported previously (48). Within these clusters, cluster 8 and cluster 30 showed expansion upon E2 treatment, whereas cluster 22 remained unchanged (Supplemental Figure 9Q). Cluster 9 was a notable exception, as it expressed markers associated with an immune-suppressive phenotype (Mrc1, Folr2, Gas6, Retnla, and Cd163; Figure 4Q and Supplemental Figure 9, M-O). This cluster also showed higher expression of $M a f$, a gene that is required for the differentiation of monocytes into macrophages (Supplemental Figure 9P). Importantly, cluster 9 showed significant expansion with E2 treatment compared with placebo (Supplemental Figure 9Q). This observation supports our hypothesis that E2 treatment leads to the expansion of macrophages that have immune-suppressive phenotypes. Taken together, this analysis suggests that E2 may promote the initial recruitment of monocytes, as evidenced by the increase in cluster 3, to the TME, where the monocytes exposed to tumor-derived factors and $\mathrm{E} 2$ underwent faster rates of differentiation and polarization to M2 macrophages (cluster 9), while at the same time suppressing expansion of M1 macrophages (cluster 15). This result was further supported by our flow cytometric data, in which we observed a trend toward an increase in the number of monocytes in response to E2 (Supplemental Figure 9R) and a decrease in M1/ $\mathrm{M} 2$ ratios, with the total number of $\mathrm{F} 480^{+}$macrophages remaining unchanged (Figures 4, A and B, and Supplemental Figure 9S).

To determine the molecular pathway or pathways that influence this M2 phenotype in E2-treated macrophages, we performed upstream regulator analysis of differentially expressed genes (DEGs) in $\mathrm{CD}^{+} 8^{+}$cells using Ingenuity Pathway Analysis (IPA). This analysis highlighted the importance of the TCF4 and WNT5A pathways (Supplemental Figure 10, A and B), the significance of which we explored in tumor-infiltrating myeloid cells 

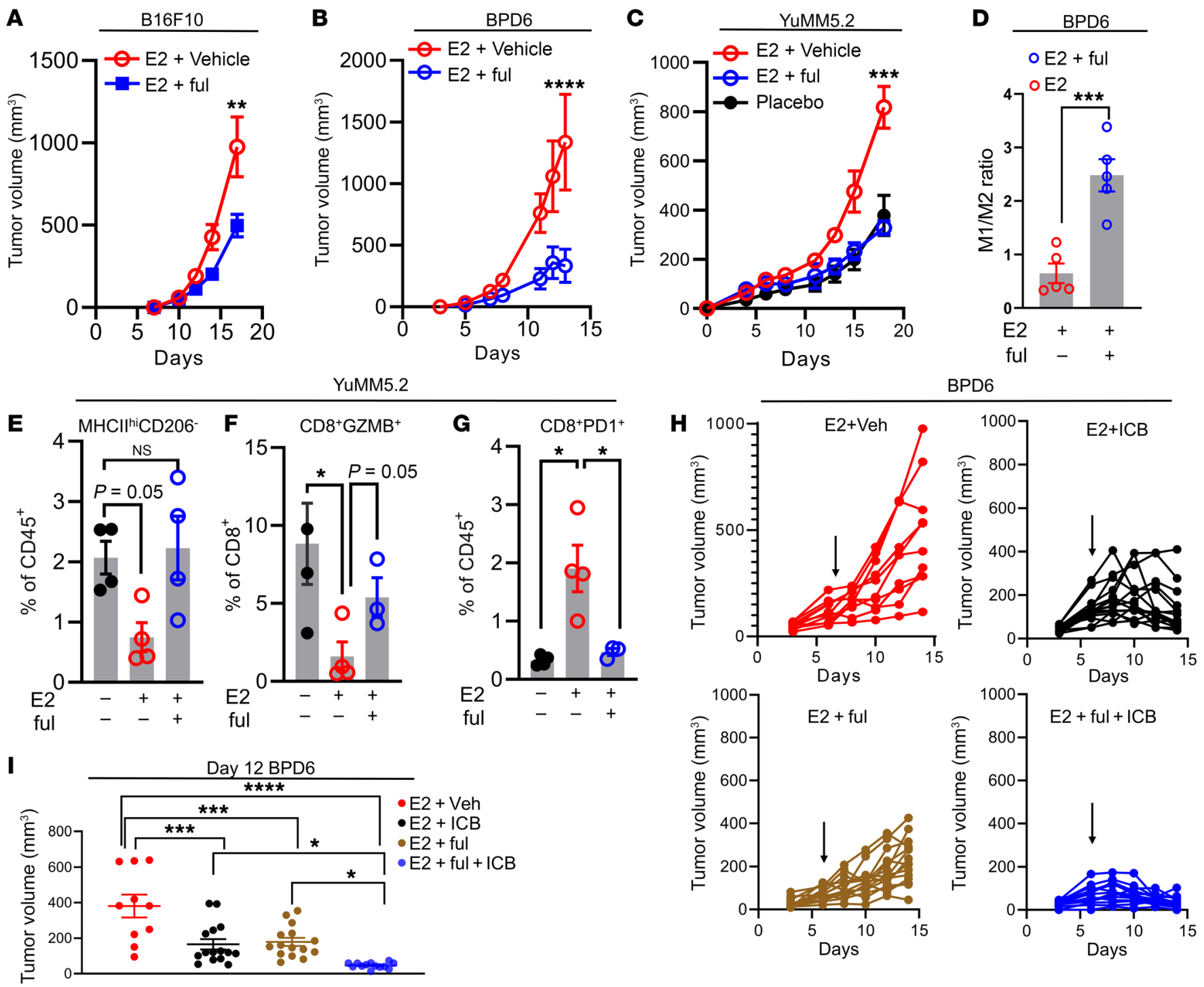

B16F10
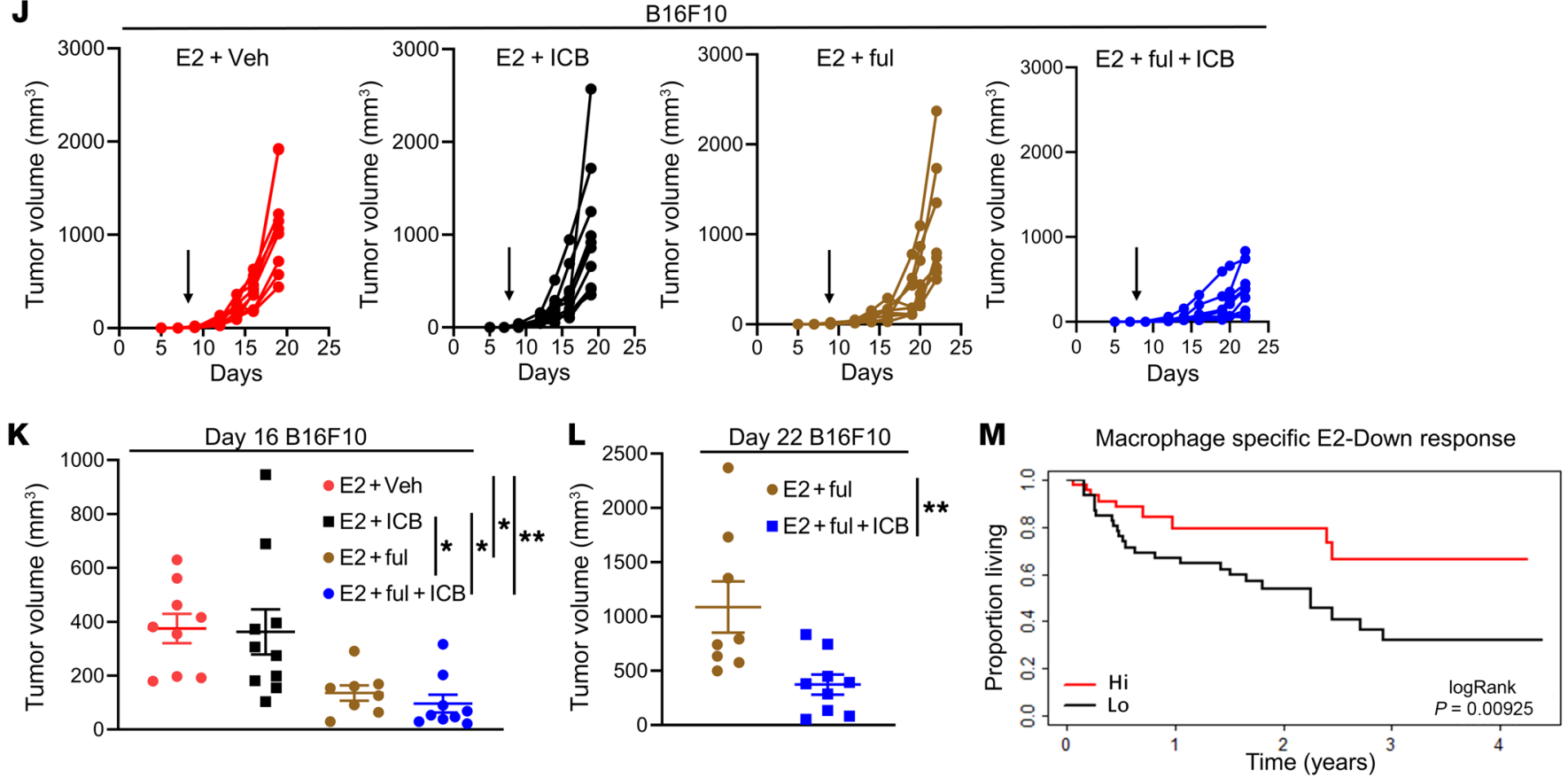
Figure 6. Pharmacological depletion of the ER reverses E2-dependent melanoma tumor growth. (A-C) Growth of B16F10 $\left(0.5 \times 10^{5}\right)(n=9)$, YuMM5.2 $\left(5 \times 10^{5}\right)(n=6)$, and BPD6 $\left(5 \times 10^{5}\right)(n=5)$ tumors in ovariectomized [57BL/6) mice supplemented with placebo or E2 and cotreated with the ER $\alpha$ antagonist fulvestrant (ful). (D) Quantification of the ratio of M1/ M2 macrophages isolated from BPD6 tumors in B. (E-G) Quantification of M1 macrophages ( $\mathrm{MHCII}^{\text {hi }} \mathrm{CD2O6}^{-}$), $\mathrm{CD}^{+} \mathrm{CZMB}^{+} \mathrm{T}$ cells and $\mathrm{CD} 8^{+} \mathrm{PD}-1^{+} \mathrm{T}$ cells in YuMM5.2 tumors from C $(n=4)$. (H) Individual volumes of BPD6 tumors implanted into ovariectomized mice treated with placebo or E2 following cotreatment with fulvestrant and ICB ( $\alpha$-PD-1 plus $\alpha$-CTLA4), either alone or in combination. Vehicle plus IgG (E2 + Veh; $n=10$; red); fulvestrant plus IgG (E2 + ful + ICB; $n=15$; blue); vehicle plus ICB (E2 + ICB; $n=15$; black); and fulvestrant plus ICB (E2 + ful; $n=15$; brown). Black arrow indicates the start of the ICB treatment regimen. (I) Tumor volumes of BPD6 measured at day 12 after inoculation. (J) Individual volumes of B16F10 tumors implanted into ovariectomized C57BL6/J mice supplemented with placebo or E2 and cotreated with fulvestrant along with ICB ( $\alpha-P D-1)$. Vehicle plus IgG ( $n=9$, red); fulvestrant plus IgC ( $n=8$, blue); vehicle plus ICB ( $n=9$, black); and fulvestrant plus ICB ( $n=10$ brown). Black arrow indicates the start of the $\alpha-P D-1$ treatment regimen. (K and $\mathbf{L}$ ) B16F10 tumor volumes measured on day 16 (all 4 groups) and day 22 (E2 plus fulvestrant vs. E2 plus fulvestrant plus $\alpha-P D-1$ groups) after inoculation. (M) Median overall survival of all patients treated with immunotherapy (pembrolizumab or nivolumab alone, or in combination with ipilimumab) from the Gide et al. data set (32), with high or low E2-downregulated gene signatures derived from $\mathrm{CD}^{+}{ }^{+}$cells in the scRNA-Seq experiments. Data in $\mathbf{A}-\mathbf{C}$ are representative of 2 individual experiments. Data are expressed as the mean \pm SEM. ${ }^{*} P<0.05,{ }^{* *} P<0.01,{ }^{* *} P<0.001$, and ${ }^{* * * *} P<0.0001$, by 2 -way ANOVA (A-C), 1-way ANOVA followed by Bonferroni's multiple-correction test (E-G, I, and $\mathbf{K}$ ), Student's $t$ test ( $\mathbf{D}$ and $\mathbf{L}$ ), and log-rank test (M).

isolated from iBP tumors excised from mice treated with placebo or E2. Gene expression analysis revealed that multiple genes in the WNT5A and TCF4 pathways were differentially regulated by E2 compared with placebo in these cells (Supplemental Figure 10C). WNT5A, signaling through the canonical $\beta$-catenin pathway, has been implicated in various biological processes including embryogenesis, cell fate development, and endothelial cell differentiation resulting in the upregulation of vasculogenic and angiogenic processes, although the significance of $\mathrm{E} 2$ in the regulation of these processes in the TME remains to be determined. Of note, WNT5A signaling has also been reported to induce tolerogenic phenotypes in macrophages in patients with breast cancer (49). We demonstrate that myeloid cells isolated from E2-treated tumors manifested a gene expression pattern characteristic of M2 macrophages, with increased expression of multiple genes, such as Vegfa, Tgm2, Tspo, and Stat1 (refs. 50-52 and Supplemental Figure 10D). It has yet to be determined whether E2-regulated expression of these genes depends on WNT signaling. In contrast to myeloid cells, knockdown of Esr1 or treatment with either E2 $(1 \mathrm{nM})$ or E2 $(1 \mathrm{nM})$ plus fulvestrant $(100 \mathrm{nM})$ did not change the expression of WNT5A/ $\beta$-catenin targets in YuMM5.2 cells (Supplemental Figure 10, E and F), although E2/ER signaling has previously been shown to influence $\beta$-catenin signaling in cancer cells (53). Together, these results indicate a likely role for $\mathrm{E} 2$ in the functional activation of WNT5A/ $\beta$-catenin signaling leading to macrophage polarization toward an immune-suppressive state in the melanoma TME.

E2 treatment suppresses antitumor $T$ cell responses. The results of the ex vivo studies described above suggest that $\mathrm{E} 2$ exerted a direct effect on macrophages to suppress the proliferation and activity of both $\mathrm{CD}^{+}$and $\mathrm{CD} 8^{+} \mathrm{T}$ cells. Flow cytometric analysis of tumor-infiltrating $\mathrm{T}$ cells from $\mathrm{iBP}$ tumors also revealed an overall decrease in the $\mathrm{CD}^{+} \mathrm{T}$ cell population with $\mathrm{E} 2$ treatment (Figure 5, $\mathrm{A}$ and $\mathrm{B}$, and Supplemental Figure 11A). Further, subgating of the $\mathrm{CD}^{+}$ $\mathrm{T}$ cell population indicated that the number of intratumoral $\mathrm{CD} 8^{+}$ cytotoxic T cells was decreased upon E2 treatment, whereas no significant change in $\mathrm{CD}_{4}{ }^{+} \mathrm{T}$ cell numbers was observed (Figure 5 , C and D, and Supplemental Figure 11, B and C). We also evaluated the activity of tumor-infiltrating $\mathrm{T}$ cells using $\mathrm{CD}^{+} \mathrm{T}$ cells isolated from syngeneic YuMM5.2 tumors. For this purpose, T cells were isolated from placebo- and E2-treated tumors and then treated ex vivo with PMA and ionomycin for 4 hours along with protein transport inhibitors. Flow cytometric analysis revealed that, when compared with $\mathrm{T}$ cells isolated from placebo-treated mice, the $\mathrm{CD} 8^{+}$ tumor-infiltrating lymphocytes (TILs) isolated from E2-treated YuMM5.2 tumors were markedly more exhausted, with significantly higher expression of PD-1 (Figure 5, E and F) and significantly reduced expression of GZMB (Figure $5, \mathrm{G}$ and $\mathrm{H}$ ), the activation markers CD44 and CD69 (Figure 5, I and J), and cytokines such as IFN- $\gamma$ (Figure $5, \mathrm{~K}$ and L). As with the iBP model, we did not observe a significant effect of $\mathrm{E} 2$ treatment on the infiltration of $\mathrm{CD}^{+}{ }^{+} \mathrm{FXP3}^{+}$Treg subsets (Supplemental Figure 11, D and E). Taken together, these results suggest that systemic E2 treatment reduced $\mathrm{CD} 8^{+} \mathrm{T}$ cell functionality, albeit in an indirect manner, as Esr1, Esr2, or Gper1 mRNAs were not expressed in T cells within the TME (Supplemental Figure 5, B-D). Further, treatment of T cells in vitro with either E2 or the SERD fulvestrant did not affect the proliferation or cytotoxic capabilities of either $\mathrm{CD} 4^{+}$or $\mathrm{CD} 8^{+}$ $\mathrm{T}$ cells (Supplemental Figure 12, A-J). Taken together, these data indicate that $\mathrm{E} 2$ indirectly reduced $\mathrm{T}$ cell function secondarily to its effects on macrophages.

Pharmacological inhibition of the ER reverses the growth-promoting effects of E2 on melanoma tumors. Fulvestrant, a SERD, acts by both inactivating and degrading the ER and is approved for use in postmenopausal patients with ER-positive breast cancer whose disease has progressed on first-line endocrine therapies (54). Fulvestrant was selected for these studies, as it is the most efficacious ER inhibitor currently available for clinical use (55). At a dose that we determined to model achievable levels in patients with breast cancer $(25 \mathrm{mg} / \mathrm{kg}$; ref. 56$)$, we found that fulvestrant significantly reduced tumor growth in all preclinical mouse models of melanoma examined (B16F10, YuMM5.2, and BPD6) (Figure 6, A-C, and Supplemental Figure 13, A-C). To understand how fulvestrant affects the TME, we analyzed the tumor-infiltrating immune cell repertoire by flow cytometry. We observed an increase in the intratumoral M1/M2 ratio or an increase in inflammatory macrophages $\left(\mathrm{MHCII}^{\mathrm{hi}} \mathrm{CD}^{206}{ }^{-}\right.$) when E2-treated mice were cotreated with fulvestrant (Figure 6, D and E, and Supplemental Figure 13, D and E). Tumor-infiltrating $\mathrm{T}$ cells from fulvestrant-treated tumors displayed an increase in cytotoxic capabilities as measured by GZMB expression (Figure 6F). Additionally, fulvestrant treatment led to a decrease in the number of $\mathrm{PD}-1^{+} \mathrm{CD} 8^{+} \mathrm{T}$ cells (exhausted $\mathrm{T}$ cells) that had increased with E2 treatment (Figure 6G). Similar observations were made in studies performed in vitro when BMDMs treated with fulvestrant were coincubated with CFSE-labeled, suboptimally activated $\left(\mathrm{CD}^{+} / \mathrm{CD} 28^{+}\right) \mathrm{T}$ cells in the presence of IL-2. Analysis of CFSE dilution revealed that the proliferation of 
T cells was not affected by their coincubation with macrophages differentiated in NM and treated with either E2 or E2 plus fulvestrant. However, exposure of T cells to macrophages, differentiated in 30\% TCM and E2, effectively suppressed $\mathrm{T}$ cell proliferation, an activity that was reversed by treatment with fulvestrant (Supplemental Figure 13F). Collectively, these results indicate that fulvestrant can inhibit the effects of E2 on tumor growth and remodel the tumor immune microenvironment to favor tumor growth inhibition in melanoma.

We next performed studies to determine whether fulvestrant could improve or restore the response to the immune checkpoint inhibitor in ICB-sensitive BPD6 and -unresponsive B16F10 tumor models. In the ICB-sensitive BPD6 model, treatment with either fulvestrant or ICB ( $\alpha$-PD-1 and $\alpha$-CTLA 4 ) slowed tumor growth, however the combination of both drugs further suppressed tumor growth when compared with each individual treatment (Figure 6, $\mathrm{H}$ and I). To determine whether fulvestrant can also increase the effectiveness of immunotherapy in the ICB-unresponsive B16F10 model, we treated mice with established B16F10 tumors with fulvestrant and $\alpha-\mathrm{PD}-1$, either alone or in combination. Importantly, the combination of fulvestrant and $\alpha-\mathrm{PD}-1$ suppressed the growth of B16F10 tumors, while P1 treatment alone had no effect (Figure 6 , J-L). Taken together, these results indicate that pharmacological targeting of $\mathrm{ER} \alpha$ can improve the intratumoral M1/M2 ratio and increase the effectiveness of ICB in both ICB-sensitive and -resistant models of melanoma. Since E2-driven tumor growth appeared to be macrophage dependent, we anticipated that a macrophage-specific ER $\alpha$ signature would predict ICB sensitivity in patients with melanoma. To this end, we first divided the E2-regulated genes in all $\mathrm{CD} 8^{+}$macrophage/monocyte clusters identified from scRNA-Seq into 2 groups: genes upregulated by E2 (E2-Up response; Supplemental File 2) and genes downregulated by E2 (E2-Down response; Supplemental File 2). We then used the human orthologs of the identified murine signatures to predict the survival of patients receiving ICB treatments using publicly available transcriptional data sets for these patients (32). We observed that an enrichment of macrophage-specific E2-downregulated genes (E2-Down) correlated with a better overall survival for patients with melanoma who had received ICB therapy (Figure $6 \mathrm{M})$. These results highlight the importance of $\mathrm{ER} \alpha$ function in TAMs residing in the melanoma TME and demonstrate how an $\mathrm{ER} \alpha$-specific signature can be used to predict a patient's response to ICB treatment.

\section{Discussion}

We have identified a tumor cell-extrinsic activity of $\mathrm{ER} \alpha$ that resulted in an increased accumulation of M2 or alternatively activated macrophages in the TME, thereby suppressing adaptive immunity and promoting tumor growth in murine models of melanoma. Previously, it has been demonstrated that E2 promotes MDSC mobilization to tumor sites and creates an immune-suppressive TME in ovarian, lung, and breast cancers (22). Although anecdotal evidence suggests that elevated numbers of circulating monocytic MDSCs track with ipilimumab treatment outcomes in patients with melanoma (57), our data reveal that it was the intratumoral M1/M2 macrophage ratio, and not changes in granulocytic MDSCs, that predicted responses in patients treated with either
PD-1 or CTLA4 alone or in combination. This encouraged us to investigate the mechanisms by which $\mathrm{E} 2$ modulates the response to ICB therapy. Here, we provide evidence that removal of endogenous estrogens (ovariectomy) provided a protective advantage against tumor growth, in part by decreasing the number of immune-suppressive TAMs and by preventing the exhaustion of cytotoxic T cells. This function was primarily attributed to E2/ER signaling in macrophages and their ability to facilitate M2 polarization. Of clinical note is the finding that the SERD fulvestrant could reverse the effects of E2 on tumor growth and the immune cell repertoire, establishing the importance of the ER in melanoma biology and highlighting a potential new treatment modality for this disease.

TAMs are one of the dominant immune cell types within the TME and can promote tumor growth by increasing neovascularization, promoting wound healing and tissue repair processes, and blocking the activation of adaptive immune cells within the TME (58-60). TAM recruitment to tumors is generally associated with resistance to chemotherapy and immunotherapy, and thus there is a high level of interest in developing interventional approaches to suppress the immune-suppressive and protumoral activities of these cells (60-63). Among the strategies used and/or under investigation are depletion of TAMs in the TME using CSF1R antibodies $(64,65)$ or bisphosphonates $(66-68)$; prevention of TAM recruitment to tumors by inhibiting the CCL2/CCR2 axis (69-71); and reprogramming of TAMs using $\alpha-\mathrm{CD} 47-\mathrm{SIRP} \alpha$ antibodies, TLR agonists, and inhibitors of the enzyme calcium calmodulin kinase kinase-2 (72-75). While somewhat successful in different tumor contexts, these therapies have often caused severe toxicities that have limited their use in patients. This highlights the potential clinical importance of our observation that estrogens (E2) can promote the establishment and maintenance of a tumor-suppressive microenvironment by TAM polarization- an activity that can be reversed by the ER antagonist/SERD fulvestrant.

Estrogens have been shown to play a major role in reducing inflammation by promoting the polarization of macrophages toward an antiinflammatory state during airway inflammation and cutaneous wound repair $(19,21)$. However, very little is known about how $\mathrm{E} 2$ affects TAM function in tumors. In breast and ovarian cancer, tumor cell-intrinsic E2/ER signaling has been linked to increased recruitment of TAMs in the TME (76-78). Our study, on the other hand, highlights a specific role for TAM-intrinsic E2/ER signaling in promoting tumor growth in validated murine models of melanoma. We have demonstrated that inhibition of estrogen action in macrophages (depletion of the ER) can recapitulate the systemic depletion of estrogen action on melanoma tumor growth. Therefore, it appears that most of the protumorigenic actions of E2 in the melanoma TME can be attributed to ER signaling in macrophages.

One of the most important findings in this study was that E2-polarized TAMs within the TME displayed the phenotypic features of M2-like immunosuppressive macrophages. This observation was confirmed by both flow cytometric analysis and pseudotime analysis of gene expression from scRNA-Seq data, in which it was revealed that E2 led to an initial accumulation of both inflammatory and patrolling monocytes. E2 then accelerated the polarization of inflammatory monocytes to M2 macrophages that 
expressed characteristic immunosuppressive markers (Cd163, Mrc1, Folr2, Retnla, and Gas6). However, the molecular mechanism or mechanisms underlying this accelerated polarization of monocytes to macrophages remain to be identified.

The functional significance of an increased accumulation of immunosuppressive macrophages was highlighted by demonstrating that E2-treated TAMs blocked the cytotoxic activity of $\mathrm{CD}^{+} \mathrm{T}$ cells by preventing GZMB expression and IFN- $\gamma$ release. Importantly, this activity was only manifested by macrophages residing in the TME and in BMDMs cultured in TCM, but not in BMDMs cultured in NM. These results indicate that soluble factors secreted by tumor cells worked in concert with E2 to promote TAM polarization, which subsequently suppressed adaptive immunity. In line with this finding, we observed changes in the expression of targets downstream of WNT5A/TCF4 signaling in tumor-associated myeloid cells treated with E2. Although functioning primarily as a positive regulator of the noncanonical WNT signaling pathway, WNT5A can in some contexts activate canonical WNT signaling through $\beta$-catenin to increase TCF/LEF transcriptional activity (79). Importantly, it has been demonstrated that tumor cell-derived WNT5A can induce $\beta$-catenin activation in DCs, leading to enhanced indoleamine 2,3-dioxygenase (IDO) production, melanoma progression, and M2 polarization (80). Since we observed E2-mediated regulation of WNT5A targets in tumor-associated myeloid cells, we speculate that tumor-derived WNT5A may work in collaboration with E2 to skew macrophage polarization toward an immune-suppressive state and suppress $\mathrm{T}$ cell activity.

In contrast to $\mathrm{CD} 8^{+} \mathrm{T}$ cells, we observed varying effects of $\mathrm{E} 2$ on $\mathrm{CD}^{+}{ }^{+} \mathrm{T}$ cell activation and/or proliferation when we cocultured these cells with macrophages in vitro versus $\mathrm{CD} 4^{+} \mathrm{T}$ cells in E2-treated tumors in vivo. Although in vitro-activated $\mathrm{CD} 4^{+} \mathrm{T}$ cells from naive mice, cocultured with myeloid cells isolated from E2-treated tumors ex vivo, showed a decrease in proliferative and cytotoxic capabilities, we detected no apparent differences in the proliferative capacity or cytotoxicity of $\mathrm{CD} 4^{+} \mathrm{T}$ cells in placeboor E2-treated tumors. Apart from TAMs, the $\mathrm{CD} 4^{+} \mathrm{T}$ cells in the tumors were chronically exposed to cytokines and factors secreted by different cell types residing in the tumor, which may account for the lack of differences in their proliferative and cytotoxic states between the placebo- and E2-treated tumors - a possibility we are currently exploring.

$\mathrm{ER} \alpha$ modulators are used as first-line treatment in $\mathrm{ER}^{+}$breast cancer, in which tumor cell-intrinsic actions of the E2/ER axis facilitate tumor growth (81). Our data demonstrate that, in hormone-resistant cancers (i.e., no direct effects of estrogens on cancer cells) such as melanoma, ER antagonists such as fulvestrant could efficiently suppress tumor growth by promoting antitumor immunity. The results of studies using tamoxifen in patients with melanoma were equivocal $(82,83)$, which is probably attributable to the drug's inherent partial ER-agonistic activity. Fulvestrant is both a high-affinity competitive antagonist and a receptor degrader that allows for a deep inhibition of ER action (84). Unfortunately, although an approved drug, its poor pharmaceutical properties have limited the clinical use of fulvestrant (85). Currently, there are 12 new orally bioavailable SERDs in clinical development, and we have an ongoing interest in evaluating the potential utility of these drugs as immune modulators. Moreover, useful cell- or process-selective ER inhibition can also be achieved with selective estrogen receptor modulators (SERMs) (i.e., bazedoxifene, lasofoxifene, and raloxifene), drugs whose relative agonist/antagonist properties differ depending on the cell/tissue context (86). Thus, in addition to profiling new SERDs, our studies provide the rationale for testing different classes of SERDs and SERMs for their ability to reprogram macrophage function and increase tumor immunity in the setting of melanoma.

One of the most important findings of this study, we believe, is that fulvestrant worked in concert with ICBs to suppress melanoma tumor growth in both ICB-sensitive and ICB-unresponsive syngeneic models of melanoma. This can be attributed, at least in part, to the ability of fulvestrant to promote a pro-immunogenic environment by elevating the M1/M2 macrophage ratio and by increasing the number of intratumoral activated $\mathrm{CD} 8^{+} \mathrm{T}$ cells. We believe this observation has significant clinical importance, as although $\alpha-\mathrm{PD}-1$ therapy is successful in some patients with melanoma, the majority of treated patients do not respond to, or acquire resistance to, this intervention. We believe that the findings in murine models of melanoma will translate to humans. This position is supported by our findings that a macrophage-derived, ER-downregulated gene signature can predict survival in patients treated with ipilimumab and pembrolizumab or nivolumab (32). These findings highlight the potential clinical utility of using a combination of ER modulators (SERDs or SERMs) with ICBs in patients with melanoma who develop ICB resistance due to an increased accumulation of immune-suppressive TAMs in tumors $(34,87)$. Additionally, we demonstrate that expression of the aromatase gene correlated with enhanced expression of TAM markers such as CD68, CSF1R, and CSF1, as well as a trend toward increased expression of PDCD1 in $\alpha-P D-1$ nonresponders. This finding suggests that, although patients who have higher levels of circulating estrogens are particularly vulnerable to developing resistance to $\alpha-\mathrm{PD}-1$ therapy, intratumoral E2 production may also contribute to disease pathobiology. One of the major side effects of ICBs is the development of immune-related adverse events, among which endocrine toxicities are most frequent. While the most common endocrinopathies related to ICB treatment is associated with thyroid dysfunction, recent reports have also suggested a significant increase in the risk of hypogonadism in ICB-treated patients $(88,89)$. Thus, the use of appropriate SERMs that demonstrate estrogenic action on reproductive organs to ameliorate the inflammatory side effects of ICB, while at the same time promoting antitumor immunity, may have added clinical utility.

In conclusion, we have demonstrated that the E2/ER axis plays an important role in macrophage reprogramming within the melanoma TME and that specific targeting of the ER signaling axis in macrophages may improve the long-term survival of patients with melanoma. Although we have provided extensive evidence showing the role of ER $\alpha$ in modulating TAM polarization and suppression of adaptive immunity, the exact mechanism or mechanisms by which $\mathrm{E} 2$ influences the immune-suppressive activity of TAMs remain to be determined. Future studies addressing the possible mechanisms by which E2 influences TAM biology will be informative as to which of the 
existing SERMs or SERDs would be most useful in ICB regimens and/or help to define the characteristics of next-generation ER modulators optimized for their positive effects on tumor immunity. Additionally, while our study was exclusively focused on TAM-intrinsic E2/ER signaling, others have shown that melanoma cells express both nuclear ERs (ER $\alpha$ and ER $\beta$; ref. 90) and GPER (16). Although the functionality of these receptors in melanoma cells is yet to be studied in detail, we cannot completely rule out the possible contribution of melanoma cell-intrinsic E2/ER signaling to the tumor growth phenotype we observed. Studies using melanoma cells genetically depleted of the ER will be informative as to the contribution of tumor cell-intrinsic E2/ ER signaling on melanoma biology.

Taken together, the results of our studies provide the underlying rationale for a clinical study we are about to undertake to explore the use of fulvestrant (and potentially other ER modulators) as a means to increase the efficacy of immune checkpoint inhibitors.

\section{Methods}

Mice. C57BL/6J, LysMCre (B6.129P2-Lyz $2^{\text {tm1(Crelffo } / J ; ~ r e f . ~ 91), ~ a n d ~}$ Pmel (B6.Cg-Thy1 a /Cy $\mathrm{Tg}$ (TcraTcrb)8Rest/J; ref. 92) mice were purchased from The Jackson Laboratory. Age-matched mice were used for all the studies. LysMCre mice were bred with Esr $1^{f l / f l}$ mice (a gift from Ken Korach, National Institute of Environmental Health Sciences [NIEHS], NIH, Durham, North Carolina, USA) to generate Esr1 $1^{f / f l}$ LysMCre and littermate control LysMCre and Esr $1^{f / f l}$ mice. iBP (Braf ${ }^{V 600 E / W T}$, Pten ${ }^{\text {fl/fl }}$ mTyrCreERT2) mice were generated by crossing breeder Braf ${ }^{W T / W T}$ Pten ${ }^{f / f l}$ mTyrCreERT2 mice with Braf ${ }^{1600 E}$ $P t e n^{f l / f l}$ mice. The mice were housed in secure animal facility cages on a 12-hour light/12-hour dark cycle at a temperature of approximately $25^{\circ} \mathrm{C}$ and $70 \%$ humidity. Mice had ad libitum access to food and water. NSG (NOD.Cg-Prkd $c^{\text {Scid }} I l 2 \mathrm{rg}^{\text {tmlwjl }} / \mathrm{SzJ}$ ) were purchased from the Division of Laboratory Animal Resources (Duke University, Durham, North Carolina, USA). The NSG animals were fed a GL3 diet and kept under pathogen-free conditions.

Tumor models and cells. The mouse B16F10 and Yumm5.2 cell lines were purchased from American Type Culture Collection (ATCC). The mouse melanoma cell line BPD6 was established from iBP as described elsewhere (80). Details on the culture conditions and tumor models are described in the Supplemental Methods.

Ovariectomy and subcutaneous pellet insertion. Ovariectomy was performed as detailed previously (93). Details on the ovariectomy procedure are discussed in Supplemental Methods.
scRNA-Seq. iBP tumors $(n=3)$ were pooled, and a single-cell suspension was isolated as described in the Supplemental Methods. Live, tumor-infiltrating immune cells $\left(\mathrm{CD}^{2} 5^{+}\right.$live-dead $\left.{ }^{-}\right)$were isolated by cell sorting and resuspended in PBS plus $0.04 \%$ BSA at a concentration of 1000 cells/ $\mu \mathrm{L}$. Details on the scRNA-Seq experiment and its analysis are provided in the Supplemental Methods.

Statistics. Statistical analysis was performed with GraphPad Prism 8.0 (GraphPad Software), using either a 2-tailed Student's $t$ test or 1- or 2-way ANOVA. For both 1-way and 2-way ANOVAs, a post-test analysis was performed using Bonferroni's multiple correction. The number of replicates is indicated in the figure legends. A $P$ value of less than 0.05 was considered statistically significant.

Study approval. All animal experiments were performed according to guidelines established and approved by the IACUC of Duke University.

Data availability. Raw data for scRNA-Seq were deposited in the NCBI's Gene Expression Omnibus (GEO) database (GEO GSE171403).

\section{Author contributions}

$\mathrm{BC}, \mathrm{CYC}$, and DPM conceived of and designed all the experiments. BC, JB, CYC, RB, WL, DM, SA, OB, KT, YB, FL, SEW, and JS performed the experimental work. $\mathrm{WG}, \mathrm{CNH}$, and $\mathrm{CMP}$ performed the analysis of scRNA-Seq data and of human correlates. BAH generated reagents, provided critical input, and helped with data interpretation. The manuscript was written by BC and DPM, with critical input from $\mathrm{CYC}$ and $\mathrm{CNH}$. The project was managed and overseen by CYC and DPM.

\section{Acknowledgments}

We thank Xia Gao for critical reading of our manuscript. We would also like to thank Prabuddha Chakraborty for helpful discussions during manuscript preparation. We would like to acknowledge the assistance of the Duke Molecular Physiology Institute Molecular Genomics core for the generation of scRNA-Seq data. This project was supported (in part) by a Melanoma Research Foundation Grant (640233); a Susan G. Komen Foundation grant (SAC180085); a Duke Cancer Institute pilot funding grant; a generous gift from Mrs. Laura Bowles Warren and Mr. James D. Warren, Esq. (to DPM); and a Susan G. Komen Foundation grant (SAC160074, to CP).

Address correspondence to: Donald P. McDonnell, Department of Pharmacology and Cancer Biology, Duke University School of Medicine, B238 LSRC, 308 Research Drive, Box 3813, Durham, North Carolina 27710, USA. Phone: 919.684.6035; Email: donald. mcdonnell@duke.edu.
1. Gogas HJ, et al. Chemotherapy for metastatic melanoma: time for a change? Cancer. 2007;109(3):455-464.

2. Chapman $\mathrm{PB}$, et al. Improved survival with vemurafenib in melanoma with BRAF V600E mutation. N Engl J Med. 2011;364(26):2507-2516.

3. Robert C, et al. Nivolumab in previously untreated melanoma without BRAF mutation. $N$ Engl J Med. 2015;372(4):320-330.

4. Topalian SL, et al. Survival, durable tumor remission, and long-term safety in patients with advanced melanoma receiving nivolumab. JClin Oncol. 2014;32(10):1020-1030.
5. Hodi FS, et al. Improved survival with ipilimumab in patients with metastatic melanoma. $N$ Engl J Med. 2010;363(8):711-723.

6. Larkin J, et al. Combined nivolumab and ipilimumab or monotherapy in untreated melanoma. N Engl J Med. 2015;373(1):23-34.

7. Sosa A, et al. Clinical assessment of immunerelated adverse events. Ther Adv Med Oncol. 2018;10:1758835918764628.

8. Sadoff $\mathrm{L}$, et al. Is malignant melanoma an endocrine-dependent tumor? The possible adverse effect of estrogen. Oncology. 1973;27(3):244-257.

9. Schmidt AN, et al. Oestrogen receptor-beta expression in melanocytic lesions. Exp Dermatol. 2006;15(12):971-980.

10. Mitkov M, et al. Steroid hormone influence on melanomagenesis. Mol Cell Endocrinol. 2015;417:94-102.

11. Moller H, et al. Recent childbirth is an adverse prognostic factor in breast cancer and melanoma, but not in Hodgkin lymphoma. Eur J Cancer. 2013;49(17):3686-3693.

12. Holly EA, et al. Cutaneous melanoma in women: ovulatory life, menopause, and use of exogenous estrogens. Cancer Epidemiol Biomarkers Prev. 1994;3(8):661-668. 
13. Huber C, et al. Antiestrogen therapy for breast cancer modifies the risk of subsequent cutaneous melanoma. Cancer Prev Res (Phila). 2012;5(1):82-88.

14. Conforti F, et al. Cancer immunotherapy efficacy and patients' sex: a systematic review and meta-analysis. Lancet Oncol. 2018;19(6):737-746.

15. Khan D, Ansar Ahmed S. The immune system is a natural target for estrogen action: opposing effects of estrogen in two prototypical autoimmune diseases. Front Immunol. 2015;6:635.

16. Natale CA, et al. Activation of $G$ protein-coupled estrogen receptor signaling inhibits melanoma and improves response to immune checkpoint blockade. Elife. 2018;7:e31770.

17. Dika E, et al. Estrogen receptors and melanoma: a review. Cells. 2019;8(11):1463.

18. Pequeux C, et al. Stromal estrogen receptor- $\alpha$ promotes tumor growth by normalizing an increased angiogenesis. Cancer Res. 2012;72(12):3010-3019.

19. Keselman A, et al. Estrogen signaling contributes to sex differences in macrophage polarization during asthma. JImmunol. 2017;199(5):1573-1583.

20. Straub RH. The complex role of estrogens in inflammation. Endocr Rev. 2007;28(5):521-574.

21. Campbell L, et al. Estrogen receptor-alpha promotes alternative macrophage activation during cutaneous repair. J Invest Dermatol. 2014;134(9):2447-2457.

22. Svoronos N, et al. Tumor cell-independent estrogen signaling drives disease progression through mobilization of myeloid-derived suppressor cells. Cancer Discov. 2017;7(1):72-85.

23. Dysthe M, Parihar R. Myeloid-derived suppressor cells in the tumor microenvironment. Adv Exp Med Biol. 2020;1224:117-140.

24. Nalbandian G, et al. The selective estrogen receptor modulators, tamoxifen and raloxifene, impair dendritic cell differentiation and activation. Jimmunol. 2005;175(4):2666-2675.

25. Pelekanou V, et al. Estrogen anti-inflammatory activity on human monocytes is mediated through cross-talk between estrogen receptor ERa36 and GPR30/GPER1. J Leukoc Biol. 2016;99(2):333-347.

26. Fujiwara T, et al. Macrophage infiltration predicts a poor prognosis for human ewing sarcoma. Am J Pathol. 2011;179(3):1157-1170.

27. Mahmoud SM, et al. Tumour-infiltrating macrophages and clinical outcome in breast cancer. J Clin Pathol. 2012;65(2):159-163.

28 . Yagi T, et al. Tumour-associated macrophages are associated with poor prognosis and programmed death ligand 1 expression in oesophageal cancer. Eur J Cancer. 2019;111:38-49.

29. Ryder M, et al. Increased density of tumor-associated macrophages is associated with decreased survival in advanced thyroid cancer. Endocr Relat Cancer. 2008;15(4):1069-1074.

30. Bronkhorst IH, et al. Detection of M2-macrophages in uveal melanoma and relation with survival. Invest Ophthalmol Vis Sci. 2011;52(2):643-650.

31. Hasita $\mathrm{H}$, et al. Significance of alternatively activated macrophages in patients with intrahepatic cholangiocarcinoma. Cancer Sci. 2010;101(8):1913-1919.

32. Gide TN, et al. Distinct immune cell populations define response to anti-PD-1 monotherapy and anti-PD-1/anti-CTLA-4 combined therapy. Cancer Cell. 2019;35(2):238-255.

33. Van Allen EM, et al. Genomic correlates of response to CTLA- 4 blockade in metastatic melanoma. Science. 2015;350(6257):207-211.

34. Hugo W, et al. Genomic and transcriptomic features of response to anti-PD-1 therapy in metastatic melanoma. Cell. 2016;165(1):35-44.

35. Charoentong $\mathrm{P}$, et al. Pan-cancer immunogenomic analyses reveal genotype-immunophenotype relationships and predictors of response to checkpoint blockade. Cell Rep. 2017;18(1):248-262.

36. Youn JI, et al. Characterization of the nature of granulocytic myeloid-derived suppressor cells in tumor-bearing mice. J Leukoc Biol. 2012;91(1):167-181.

37. Schlecker E, et al. Tumor-infiltrating monocytic myeloid-derived suppressor cells mediate CCR5-dependent recruitment of regulatory $\mathrm{T}$ cells favoring tumor growth. J Immunol. 2012;189(12):5602-5611.

38. Bindea G, et al. Spatiotemporal dynamics of intratumoral immune cells reveal the immune landscape in human cancer. Immunity. 2013;39(4):782-795.

39. Newman AM, et al. Robust enumeration of cell subsets from tissue expression profiles. Nat Methods. 2015;12(5):453-457.

40. Ye Y, et al. Sex-associated molecular differences for cancer immunotherapy. Nat Commun. 2020;11(1):1779.

41. Marzagalli M, et al. Estrogen receptor $\beta$ in melanoma: from molecular insights to potential clinical utility. Front Endocrinol (Lausanne). 2016;7:140.

42. Rajabi P, et al. Expression of estrogen receptor alpha in malignant melanoma. Adv Biomed Res. 2017;6:14.

43. Dankort D, et al. Braf(V600E) cooperates with Pten loss to induce metastatic melanoma. Nature Genet. 2009;41(5):544-552.

44. Kohler C, et al. Mouse cutaneous melanoma induced by mutant BRaf arises from expansion and dedifferentiation of mature pigmented melanocytes. Cell Stem Cell. 2017;21(5):679-693.

45. Benner B, et al. Generation of monocyte-derived tumor-associated macrophages using tumor-conditioned media provides a novel method to study tumor-associated macrophages in vitro. JImmunother Cancer. 2019;7(1):140.

46. Lavin Y, et al. Innate immune landscape in early lung adenocarcinoma by paired single-cell analyses. Cell. 2017;169(4):750-765.

47. Yang J, et al. Monocyte and macrophage differentiation: circulation inflammatory monocyte as biomarker for inflammatory diseases. Biomark Res. 2014;2(1):1.

48. Trombetta AC, et al. A circulating cell population showing both M1 and M2 monocyte/macrophage surface markers characterizes systemic sclerosis patients with lung involvement. Respir Res. 2018;19(1):186

49. Bergenfelz C, et al. Wnt5a induces a tolerogenic phenotype of macrophages in sepsis and breast cancer patients. J Immunol. 2012;188(11):5448-5458.

50. Yang P, et al. TGM2 interference regulates the angiogenesis and apoptosis of colorectal cancer via Wnt/ $\beta$-catenin pathway. Cell Cycle. 2019;18(10):1122-1134.

51. Wolf A, et al. The TSPO-NOX1 axis controls phagocyte-triggered pathological angiogenesis in the eye. Nat Commun. 2020;11(1):2709.

52. Battle TE, et al. Signal transducer and activator of transcription 1 activation in endothelial cells is a negative regulator of angiogenesis. Cancer Res. 2006;66(7):3649-3657.

53. Liu S, et al. Estrogen receptor alpha regulates the Wnt/ $\beta$-catenin signaling pathway in colon cancer by targeting the NOD-like receptors. Cell Signal. 2019;61:86-92.

54. Nathan MR, Schmid P. A review of fulvestrant in breast cancer. Oncol Ther. 2017;5(1):17-29.

55 . Robertson JF, et al. Activity of fulvestrant $500 \mathrm{mg}$ versus anastrozole $1 \mathrm{mg}$ as first-line treatment for advanced breast cancer: results from the FIRST study. J Clin Oncol. 2009;27(27):4530-4535.

56. Wardell SE, et al. Pharmacokinetic and pharmacodynamic analysis of fulvestrant in preclinical models of breast cancer to assess the importance of its estrogen receptor-alpha degrader activity in antitumor efficacy. Breast Cancer Res Treat. 2020;179(1):67-77.

57. Meyer C, et al. Frequencies of circulating MDSC correlate with clinical outcome of melanoma patients treated with ipilimumab. Cancer Immunol Immunother. 2014;63(3):247-257.

58. Ostuni R, et al. Macrophages and cancer: from mechanisms to therapeutic implications. Trends Immunol. 2015;36(4):229-239.

59. Cassetta L, Pollard JW. Targeting macrophages: therapeutic approaches in cancer. Nat Rev Drug Discov. 2018;17(12):887-904.

60. Petty AJ, Yang Y. Tumor-associated macrophages: implications in cancer immunotherapy. Immunotherapy. 2017;9(3):289-302.

61. DeNardo DG, et al. Leukocyte complexity predicts breast cancer survival and functionally regulates response to chemotherapy. Cancer Discov. 2011;1(1):54-67.

62. Arlauckas SP, et al. In vivo imaging reveals a tumor-associated macrophage-mediated resistance pathway in anti-PD-1 therapy. Sci Transl Med. 2017;9(389):eaal3604.

63. Gordon SR, et al. PD-1 expression by tumour-associated macrophages inhibits phagocytosis and tumour immunity. Nature. 2017;545(7655):495-499.

64. Tap WD, et al. Structure-guided blockade of csf1 kinase in tenosynovial giant-cell tumor. $N$ Engl J Med. 2015;373(5):428-437.

65. Butowski N, et al. Orally administered colony stimulating factor 1 receptor inhibitor PLX3397 in recurrent glioblastoma: an Ivy Foundation Early Phase Clinical Trials Consortium phase II study. Neuro Oncol. 2016;18(4):557-564.

66. Audic Y, Hartley RS. Post-transcriptional regulation in cancer. Biol Cell. 2004;96(7):479-498.

67. Ben-Aharon I, et al. Bisphosphonates in the adjuvant setting of breast cancer therapy - effect on survival: a systematic review and meta-analysis. PLoS One. 2013;8(8):e70044.

68. Gnant M, et al. Endocrine therapy plus zoledronic acid in premenopausal breast cancer. $N$ Engl J Med. 2009;360(7):679-6791.

69. Sandhu SK, et al. A first-in-human, first-in-class, phase I study of carlumab (CNTO 888), a human monoclonal antibody against CC-chemokine ligand 2 in patients with solid tumors. Cancer Chemother Pharmacol. 2013;71(4):1041-1050.

70. Pienta KJ, et al. Phase 2 study of carlumab (CNTO 
888), a human monoclonal antibody against CC-chemokine ligand 2 (CCL2), in metastatic castration-resistant prostate cancer. Invest New Drugs. 2013;31(3):760-768.

71. Nywening TM, et al. Targeting tumour-associated macrophages with CCR2 inhibition in combination with FOLFIRINOX in patients with borderline resectable and locally advanced pancreatic cancer: a single-centre, open-label, dose-finding, non-randomised, phase $1 \mathrm{~b}$ trial. Lancet Oncol. 2016;17(5):651-662.

72. Kobold S, et al. Modes of action of TLR7 agonists in cancer therapy. Immunotherapy. 2014;6(10):1085-1095.

73. Chao MP, et al. Anti-CD47 antibody synergizes with rituximab to promote phagocytosis and eradicate non-Hodgkin lymphoma. Cell. 2010;142(5):699-713.

74. Liu J, et al. Pre-clinical development of a humanized anti-CD47 antibody with anti-cancer therapeutic potential. PLoS One. 2015;10(9):e0137345.

75. Racioppi L, et al. CaMKK2 in myeloid cells is a key regulator of the immune-suppressive microenvironment in breast cancer. Nat Commun. 2019;10(1):2450.

76. Svensson S, et al. CCL2 and CCL5 are novel therapeutic targets for estrogen-dependent breast cancer. Clin Cancer Res. 2015;21(16):3794-3805.

77. Ciucci A, et al. Multiple direct and indirect mechanisms drive estrogen-induced tumor growth in high grade serous ovarian cancers. Oncotarget. 2016;7(7):8155-8171.

78. Ning C, et al. Infiltrating macrophages induce ER $\alpha$ expression through an IL17A-mediated epigenetic mechanism to sensitize endometrial cancer cells to estrogen. Cancer Res. 2016;76(6):1354-1366.

79. Okamoto M, et al. Noncanonical Wnt5a enhances Wnt/ $\beta$-catenin signaling during osteoblastogenesis. Sci Rep. 2014;4:4493.

80. Zhao F, et al. Paracrine Wnt5a- $\beta$-catenin signaling triggers a metabolic program that drives dendritic cell tolerization. Immunity. 2018;48(1):147-160.

81. Haines $\mathrm{CN}$, et al. Current and emerging estrogen receptor-targeted therapies for the treatment of breast cancer [published online July 30, 2021]. Essays Biochem. https://doi.org/10.1042/ ebc20200174.

82. Cocconi G, et al. Treatment of metastatic malignant melanoma with dacarbazine plus tamoxifen. N EnglJMed.1992;327(8):516-523.

83. Lens MB, et al. Use of tamoxifen in the treatment of malignant melanoma. Cancer. 2003;98(7):1355-1361.

84. Wardell SE, et al. The turnover of estrogen receptor $\alpha$ by the selective estrogen receptor degrader (SERD) fulvestrant is a saturable process that is not required for antagonist efficacy. Biochem Pharmacol. 2011;82(2):122-130.

85. McDonnell DP, et al. Next-generation endo- crine therapies for breast cancer. JClin Oncol. 2021;39(12):1383-1388.

86. Martinkovich S, et al. Selective estrogen receptor modulators: tissue specificity and clinical utility. Clin Interv Aging. 2014;9:1437-1452.

87. Neubert NJ, et al. T cell-induced CSF1 promotes melanoma resistance to PD1 blockade. Sci Transl Med. 2018;10(436):eaan3311.

88. Bai X, et al. Mapping endocrine toxicity spectrum of immune checkpoint inhibitors: a disproportionality analysis using the WHO adverse drug reaction database, VigiBase. Endocrine. 2020;69(3):670-681.

89. Ozdemir BC. Immune checkpoint inhibitor-related hypogonadism and infertility: a neglected issue in immuno-oncology. J Immunother Cancer. 2021;9(2):e002220.

90. Ohata C, et al. Expression of estrogen receptor beta in normal skin, melanocytic nevi and malignant melanomas. J Dermatol. 2008;35(4):215-221.

91. Clausen BE, et al. Conditional gene targeting in macrophages and granulocytes using LysMcre mice. Transgenic Res. 1999;8(4):265-277.

92. Overwijk WW, et al. Tumor regression and autoimmunity after reversal of a functionally tolerant state of self-reactive CD8+ T cells. JExp Med. 2003;198(4):569-580.

93. Nelson ER, et al. 27-Hydroxycholesterol links hypercholesterolemia and breast cancer pathophysiology. Science. 2013;342(6162):1094-1098. 